\title{
On the characterization of infinitesimal symmetries of the relativistic phase space
}

\author{
Josef Janyška ${ }^{1}$, Raffaele Vitolo ${ }^{2}$ \\ ${ }^{1}$ Department of Mathematics and Statistics, Masaryk University \\ Kotlářská 2, 61137 Brno, Czech Republic \\ email: janyska@math.muni.cz \\ ${ }^{2}$ Department of Mathematics and Physics "E. De Giorgi", University of Lecce \\ Via per Arnesano, 73100 Lecce, Italy \\ email: raffaele.vitolo@unisalento.it \\ Published in Journal of Physics A: Math. Theor., \\ Vol. 45 (2012) 485205 (28pp).
}

\begin{abstract}
The phase space of relativistic particle mechanics is defined as the 1st jet space of motions regarded as timelike 1-dimensional submanifolds of spacetime. A Lorentzian metric and an electromagnetic 2 -form define naturally on the odd-dimensional phase space a generalized contact structure. In the paper infinitesimal symmetries of the phase structures are characterized. More precisely, it is proved that all phase infinitesimal symmetries are special Hamiltonian lifts of distinguished conserved quantities on the phase space. It is proved that generators of infinitesimal symmetries constitute a Lie algebra with respect to a special bracket. A momentum map for groups of symmetries of the geometric structures is provided.

Key words: Relativistic mechanics, jets of submanifolds, nonlinear connections, contact forms, cosymplectic forms, infinitesimal symmetries.
\end{abstract}

MSC2000: 70H40,70H45,70H33,70G45,58A20. 


\section{Introduction}

Relativistic mechanics of particles with mass is usually formulated, in a differential geometric context, as a theory on the tangent bundle of spacetime endowed with the symplectic form induced by the pseudo-Riemannian metric. Anyway, this formulation has the drawback of presenting a degenerate Lagrangian. The degeneracy is due to the fact that the Lagrangian is invariant with respect to the affine reparametrization of curves, which acts as a gauge group of the theory [8, 10. As a way to solve it, some authors tried to formulate mechanics on 'trajectories', or non-parametrized curves [28, 34]. None of them, however, present a complete model for the relativistic mechanics.

Recently, a formulation of relativistic mechanics based on jets of submanifolds and contact forms has been presented [14, 16]. Here the tangent bundle is replaced by the first jet space of time-like curves as the phase space of the theory. Time-like curves can be regarded as distinguished 1dimensional submanifolds: indeed, the phase space is a subspace of the first jet of 1-dimensional submanifolds of spacetime.

The literature on jets of submanifolds (also known as 'contact elements', 'differentiable elements', 'first-order caps', 'extended jets' etc., see [2, 21, 29] and references therein) is less spread with respect to the literature on jets of fibrings. Jets of 1-dimensional submanifolds of order $r$ are equivalence classes of 1-dimensional submanifolds having a contact of order $r$. Clearly, two curves have a contact if and only if their tangent space is the same; hence this notion is independent of the parametrization. In particular, the phase space of relativistic mechanics is the set of first jets of time-like curves, and in the case of vanishing electromagnetic field, the equation of motion is the equation for unparametrized geodesics, or geodesics as 1-dimensional submanifolds. Reparametrization-invariant theories are quite common (e.g., string theories, [8]) and it is always possible to get rid of reparametrization invariance without any gauge-fixing by using jets of submanifolds, which can be regarded as ordinary jets factored by the reparametrization group [21].

In [14, 16, 17] a structure generalizing contact and cosymplectic structures (in the sense of [1, 6, 17, 22, 23]) naturally induced from the gravitational and the electromagnetic fields has been introduced on the first jet space of timelike curves. Such a structure is a special case of generalized contact structure, [31, 37], that we call almost-cosymplectic-contact structure. The structure has a one-dimensional kernel (Reeb vector field) whose integral curves are the trajectories of the system. A non-degenerate Lagrangian formulation of the theory was developed in [24] using the variational calculus on jets of submanifolds [5, 25, 35. Indeed, our model is a universal mass shell, i.e. it is isomorphic to a mass shell [10] for each choice of a particle mass. 
In Dirac's languange, we work on a primary constraint which is naturally endowed with a generalized contact structure. A Hamiltonian formulation is straightforward in this context.

Then, a quantum theory for a scalar particle on the above background has been formulated by analogy with the geometric quantization of classical mechanical systems [36], and this leads to a covariant Klein-Gordon equation [12].

In this paper we study infinitesimal symmetries of the geometrical structures which are naturally given on the phase space by a pseudo-Riemannian metric $g$ and an electromagnetic field $F$.

The study of such infinitesimal symmetries dates back to [11, where the phase space was taken to be the (odd-dimensional) pseudosphere subbundle of the tangent bundle of spacetime. The geometrical structure of such phase space is obtained as the restriction of the canonical symplectic structure given on the tangent bundle of the spacetime by $g$ and $F$. It can be easily realized that the 1-jet of time-like submanifolds and the pseudosphere subbundle are isomorphic, but the advantage of jet space over pseudosphere is that, while the pseudosphere is defined by an equation, there exists on the jet space a natural choice of coordinates induced by spacetime coordinates. This makes the computations much easier. The main result in [11] is that the tangent lift of a vector field $X$ on $\boldsymbol{E}$ is an infinitesimal symmetry of the phase structure if and only if $X$ is an infinitesimal symmetry of $g$ and $F$, i.e. $L_{X} g=0$ and $L_{X} F=0$. Moreover, conserved quantities related with the above symmetries are described.

In our approach to the phase space we obtain results which are "parallel" to results for Galilean spacetime in [19, 26, 32]. Namely, we prove that all projectable infinitesimal symmetries of the generalized contact structure are holonomic lifts to the phase space of infinitesimal symmetries of $g$ and $F$. Here, "projectable" means that we consider infinitesimal symmetries that project onto vector fields on spacetime, and "holonomic lift" means the lift of a vector field to the first jet space.

Moreover, we prove that all such infinitesimal symmetries can be obtained as special Hamiltonian lifts (i.e., lifts defined through the generalized contact structure) of distinguished functions on the phase space, which we call special phase functions. Special phase functions are uniquely determined by a spacetime vector field and a function on spacetime, and have the form defined by eq. 1. In the case when they generate an infinitesimal symmetry of the generalized contact structure, the vector field is a symmetry of $g$ and $F$ and the special phase function is a conserved quantity. Another new feature of our approach is that the sheaf of generators of infinitesimal symmetries constitute a Lie algebra with respect to the special bracket (i.e., a bracket 
defined through the generalized contact structure) of special phase functions.

The above infinitesimal symmetries turn out to be Noether symmetries of the Lagrangian, hence they are symmetries of the Euler-Lagrange morphism. This implies that they are point symmetries of the Euler-Lagrange equation, altough they do not exhaust that class, see below.

Finally, by analogy with the standard momentum map for Hamiltonian systems (see, for example, [30]), we provide an equivariant momentum map for the generalized contact structure. This will be the starting point for a Marsden-Weinstein type reduction of the phase space, in the spirit of the general theory exposed in [1, 4, 6]. As far as we know, this has never been attempted in relativistic mechanics, and will be the subject of future work.

As a last comment, we are aware of the fact that the class of symmetries that we considered in this paper can be substantially enlarged. We do not consider generalizations to the computation of point or generalized symmetries of the equation of motion since they would not be symmetries of the geometric structures on the phase space like the generalized contact structure. The most important generalization is obtained by dropping the projectability hypothesis and dealing with vector fields depending on the phase space in an essential way. Non-projectable symmetries of this type are called higher symmetries [3], generalized symmetries [29] or hidden symmetries (see, for example, [33]) because they are not related to any symmetry of spacetime. Non-projectable symmetries of our generalized contact structure would be Noether symmetries of the equation of motion, like proper affine or projective symmetries [9], and would correspond to conserved quantities which can be higher degree polynomials in velocities. In this case, coefficients have tensor character and constitute the so-called Killing tensors. Killing-Yano tensors also play an analogous role in defining hidden symmetries.

Our plan was to focus our attention to symmetries in the context of the generalized contact geometry of general relativistic spacetime. On the other hand, as a future research plan it would be interesting to characterize nonprojectable symmetries of the geometric structures on the phase space. At the present moment, we cannot predict what are the results of the paper that could be extended to more general symmetries. In particular, we do not know if it would be possible to define a momentum map, or to associate a wider class of special phase functions and a Lie bracket between them. See also the discussion in Section 5 .

Acknowledgements. This work has been partially supported by the grant GA ČR 201/09/0981, by the Istituto Nazionale di Alta Matematica and the Dipartimento di Matematica e Fisica "E. De Giorgi". We thank Marco Modugno for many useful discussions. We also thank the anonymous 
referees for many useful remarks that helped us to improve the presentation of our results.

\section{Preliminaries}

We assume manifolds and maps to be $\mathcal{C}^{\infty}$.

Unit spaces. The theory of unit space has been developed in [20] in order to make explicit the independence of classical and quantum mechanics from the choice of unit of measurements. Unit spaces have the same algebraic structure as $\mathbb{R}_{+}$, but no natural basis. We assume the (1-dimensional) unit spaces $\mathbb{T}$ (space of time intervals), $\mathbb{L}$ (space of lengths) and $\mathbb{M}$ (space of masses). We set $\mathbb{T}^{-1} \equiv \mathbb{T}^{*}$, and analogously for $\mathbb{L}, \mathbb{M}$. Tensor fields appearing in the theory will be usually scaled, i.e., they will take values in unit spaces according to their physical interpretation. For example, the metric will take values in the space of area units $\mathbb{L}^{2}$.

We assume the following constant elements: the light velocity $c \in \mathbb{T}^{-1} \otimes \mathbb{L}$ and the Planck's constant $\hbar \in \mathbb{T}^{-1} \otimes \mathbb{L}^{2} \otimes \mathbb{M}$. Moreover, we say a charge to be an element $q \in \mathbb{T}^{-1} \otimes \mathbb{L}^{3 / 2} \otimes \mathbb{M}^{1 / 2} \otimes \mathbb{R}$.

We will assume coordinates to be dimensionless (i.e., real valued).

Generalized Lie derivatives. We will use a general approach to Lie derivatives [21, p. 376]. If $f: \boldsymbol{M} \rightarrow \boldsymbol{N}$ is a mapping, $X$ a vector field on $\boldsymbol{M}$ and $Y$ a vector field on $\boldsymbol{N}$ then we define Lie derivative of $f$ with respect to the pair $(X, Y)$ as the mapping

$$
L_{(X, Y)} f=T f \circ X-Y \circ f: M \rightarrow T \boldsymbol{N} .
$$

This definition includes the standard definition of Lie derivative as a special case. See [21, p. 59] for more details.

Infinitesimal symmetries of geometrical structures of odd dimensional manifolds. Let $\boldsymbol{M}$ be a $(2 n+1)$-dimensional manifold. A pre cosymplectic (regular) structure (pair) on $\boldsymbol{M}$ is given by a 1 -form $\omega$ and a 2-form $\Omega$ such that $\omega \wedge \Omega^{n} \not \equiv 0$. A contravariant (regular) structure (pair) $(E, \Lambda)$ is given by a vector field $E$ and a 2 -vector $\Lambda$ such that $E \wedge \Lambda^{n} \not \equiv 0$. We denote by $\Omega^{b}: T \boldsymbol{M} \rightarrow T^{*} \boldsymbol{M}$ and $\Lambda^{\sharp}: T^{*} \boldsymbol{M} \rightarrow T \boldsymbol{M}$ the corresponding "musical" morphisms. By $[23]$ if $(\omega, \Omega)$ is a pre cosymplectic pair then there exists a unique regular pair $(E, \Lambda)$ such that

$$
\left(\Omega_{\mid \operatorname{im} \Lambda^{\sharp}}\right)^{-1}=\Lambda_{\mid \operatorname{im} \Omega^{b}}^{\sharp}, \quad i_{E} \omega=1, \quad i_{E} \Omega=0, \quad i_{\omega} \Lambda=0 .
$$

On the other hand for any regular pair $(E, \Lambda)$ there exists a unique (regular) pair $(\omega, \Omega)$ satisfying the above identities. The pairs $(\omega, \Omega)$ and $(E, \Lambda)$ 
satisfying the above identities are said to be mutually dual. The vector field $E$ is usually called the Reeb vector field of $(\omega, \Omega)$. A vector field $X$ on $\boldsymbol{M}$ is said to be $\omega$-vertical if and only if $i_{X} \omega=0$ and $E$-horizontal if and only if $i_{X} \Omega=0$. Any vector field can be decomposed in a unique way as the sum of $E$-horizontal and $\omega$-vertical parts. Similarly a 1 -form $\alpha$ on $\boldsymbol{M}$ is said to be $E$-vertical if and only if $i_{\alpha} E=0$ and $\omega$-horizontal if and only if $i_{\alpha} \Lambda=0$. Any 1 -form can be decomposed in a unique way as the sum of $\omega$-horizontal and $E$-vertical parts.

Let $(\omega, \Omega)$ and $(E, \Lambda)$ be mutually dual regular structures on $\boldsymbol{M}$. An infinitesimal symmetry of the structure $(\omega, \Omega)$ is a vector field $X$ on $\boldsymbol{M}$ such that $L_{X} \omega=0, L_{X} \Omega=0$. Similarly, an infinitesimal symmetry of the structure $(E, \Lambda)$ is a vector field $X$ on $\boldsymbol{M}$ such that $L_{X} E=[X, E]=0$, $L_{X} \Lambda=[X, \Lambda]=0$.

1.1 Lemma. Let $X$ be a vector field on $\boldsymbol{M}$. The following conditions are equivalent:

1. $L_{X} \omega=0$ and $L_{X} \Omega=0$.

2. $L_{X} E=[X, E]=0$ and $L_{X} \Lambda=[X, \Lambda]=0$.

Proof. For the dual structures we have the identities (1). Then we get the identities

$$
\begin{array}{ll}
i_{L_{X} \Lambda} \Omega+i_{\Lambda} L_{X} \Omega=0, & i_{L_{X} E} \omega+i_{E} L_{X} \omega=0, \\
i_{L_{X} E} \Omega+i_{E} L_{X} \Omega=0, & i_{L_{X} \omega} \Lambda+i_{\omega} L_{X} \Lambda=0 .
\end{array}
$$

Suppose that $L_{X} \omega=0$ and $L_{X} \Omega=0$. Then $i_{L_{X} E} \Omega=0$ implies that $E$-horizontal part of $L_{X} E$ vanishes, and $i_{L_{X} E} \omega=0$ implies that $\omega$-vertical part of $L_{X} E$ vanishes. So, $L_{X} E=0$.

We have, [17, $\Omega^{\mathrm{b}} \circ \Lambda^{\sharp}=\mathrm{id}_{T^{*} M}-E \otimes \omega$ which implies

$$
\left(L_{X} \Omega\right)^{b} \circ \Lambda^{\sharp}+\Omega^{b} \circ\left(L_{X} \Lambda\right)^{\sharp}=-L_{X} E \otimes \omega-E \otimes L_{X} \omega .
$$

So, $\Omega^{b} \circ\left(L_{X} \Lambda\right)^{\sharp}=0$ which implies that $E$-horizontal part of $\left(L_{X} \Lambda\right)^{\sharp}(\alpha)$ vanishes for any 1 -form $\alpha$. Further we have $i_{\omega} i_{\alpha} L_{X} \Lambda=0$. Really,

$$
\begin{aligned}
i_{\omega} i_{\alpha} L_{X} \Lambda= & i_{\alpha \wedge \omega}[X, \Lambda]=i_{[X, \Lambda]}(\alpha \wedge \omega) \\
= & i_{X} d i_{\Lambda}(\alpha \wedge \omega)-i_{\Lambda} d i_{X}(\alpha \wedge \omega)-i_{X \wedge \Lambda} d(\alpha \wedge \omega) \\
= & -i_{\Lambda}[d(\omega(X)) \wedge \alpha+\omega(X) d \alpha-d(\alpha(X)) \wedge \omega-\alpha(X) d \omega \\
& \left.+\left(i_{X} d \omega\right) \wedge \alpha+\alpha(X) d \omega-\omega(X) d \alpha+\omega \wedge\left(i_{X} d \alpha\right)\right] \\
= & -i_{\Lambda}\left[\left(d i_{X} \omega+i_{X} d \omega\right) \wedge \alpha\right]=-i_{\Lambda}\left(L_{X} \omega \wedge \alpha\right)=0 .
\end{aligned}
$$

So, we get that $\omega$-vertical part of $\left(L_{X} \Lambda\right)^{\sharp}(\alpha)$ vanishes for any $\alpha$ and $\left(L_{X} \Lambda\right)^{\sharp}(\alpha)=0$. Then $L_{X} \Lambda=0$.

The opposite implication can be proved in the same manner.

QED

In differential geometry of odd-dimensional manifolds two types of geometrical structures are usually considered: a cosymplectic structure [1, 6, 7, 
22 is defined by a cosymplectic pair $(\omega, \Omega)$, where $\omega$ is a 1 -form and $\Omega$ a 2 -form, such that $d \omega=0, d \Omega=0, \omega \wedge \Omega^{n} \not \equiv 0$, and a contact structure [22, pag. 285] is defined by a contact pair $(\omega, \Omega)$, where $\omega$ is a $1-$ form and $\Omega$ a 2 -form, such that $\Omega=d \omega, \omega \wedge \Omega^{n} \not \equiv 0$.

More generally, we define an almost-cosymplectic-contact structure [17] by a regular almost-cosymplectic-contact pair $(\omega, \Omega)$, where $\omega$ is a 1 -form and $\Omega$ a $2-$ form, such that

$$
d \Omega=0, \quad \omega \wedge \Omega^{n} \not \equiv 0 .
$$

Thus, an almost-cosymplectic-contact structure becomes a cosymplectic structure when $d \omega=0$ and a contact structure when $\Omega=d \omega$.

We recall that a Jacobi structure [22, pag. 337] is defined by a Jacobi pair $(E, \Lambda)$, where $E$ is a vector field and $\Lambda$ a 2 -vector, such that $[E, \Lambda]=0$, $[\Lambda, \Lambda]=-2 E \wedge \Lambda$, where [,] denotes the Schouten bracket. Further, a coPoisson structure [17] is defined by a pair $(E, \Lambda)$ such that $[E, \Lambda]=0$, $[\Lambda, \Lambda]=0$.

More generally, we define an almost-coPoisson-Jacobi structure [17] by an almost-coPoisson-Jacobi pair $(E, \Lambda)$, along with a fundamental 1-form $\omega$ such that

$$
[E, \Lambda]=-E \wedge \Lambda^{\sharp}\left(L_{E} \omega\right), \quad[\Lambda, \Lambda]=2 E \wedge\left(\left(\Lambda^{\sharp} \otimes \Lambda^{\sharp}\right)(d \omega)\right) .
$$

We have

1.2 Theorem. [17] Let $(\omega, \Omega)$ be a regular pair and $(E, \Lambda)$ its dual regular pair. Then,

(1) $(\omega, \Omega)$ is an almost-cosymplectic-contact pair if and only if $(E, \Lambda)$ is an almost-coPoisson-Jacobi pair along with the fundamental 1-form $\omega$;

(2) $(\omega, \Omega)$ is a cosymplectic pair if and only if $(E, \Lambda)$ is a coPoisson pair;

(3) $(\omega, \Omega)$ is a contact pair if and only if $(E, \Lambda)$ is a Jacobi pair.

Note that all the above mentioned covariant and their dual contravariant structures define generalized contact structures on $T \boldsymbol{M} \oplus T^{*} \boldsymbol{M}$ in the sense of [31, 37].

Jets of submanifolds. Here we will recall the basics about jets of 1dimensional submanifolds. Our main sources are [2, 25, 27], where the structures constructed below are given in the general case of jets of submanifolds of arbitrary dimension.

Let $\boldsymbol{E}$ be a manifold, and let $\operatorname{dim} \boldsymbol{E}=1+m$. Let $r \geq 0$. An $r$-jet of a 1-dimensional submanifold $s: \boldsymbol{S} \subset \boldsymbol{E}$ at $x \in \boldsymbol{E}$ is defined to be the 
equivalence class of 1-dimensional submanifolds having a contact with $s$ of order $r$ at $x$. The equivalence class is denoted by $j_{r} s(x)$, and the set of all $r$ jets of all 1-dimensional submanifolds at $x \in \boldsymbol{E}$ is denoted by $J_{r x}(\boldsymbol{E}, 1)$. The set $J_{r}(\boldsymbol{E}, 1)=\sqcup_{x \in \boldsymbol{E}} J_{r x}(\boldsymbol{E}, 1)$ is said to be the $r$-jet space of 1-dimensional submanifolds of $\boldsymbol{E}$. Of course we have $J_{0}(\boldsymbol{E}, 1)=\boldsymbol{E}$. For $r>p$ we have the projections $\pi_{p}^{r}: J_{r}(\boldsymbol{E}, 1) \rightarrow J_{p}(\boldsymbol{E}, 1)$.

A chart $\left(x^{\lambda}\right)$ on $\boldsymbol{E}$ is said to be divided if its coordinates are divided in two subsets of 1 and $m$ elements. In what follows Greek indices $\lambda, \mu, \ldots$ will run from 0 to $m$, and will label coordinates on $\boldsymbol{E}$, while Latin indices $i, j, \ldots$ will run from 1 to $m$. We will use the notation $\left(x^{0}, x^{i}\right)$ for divided charts. We denote by $\left(\partial_{\lambda}\right)$ and $\left(d^{\lambda}\right)$ the local basis of vector fields and 1-forms induced by a chart $\left(x^{\lambda}\right)$.

A divided chart $\left(x^{0}, x^{i}\right)$ on $\boldsymbol{E}$ is said to be adapted to a 1-dimensional submanifold $s: \boldsymbol{S} \subset \boldsymbol{E}$ if $s$ can be expressed in coordinates as $\left(\breve{x}^{0}, s^{i}\right)$, where $\breve{x}^{0}:=\left.x^{0}\right|_{S}$ and $s^{i}:=x^{i} \circ s \circ\left(\breve{x}^{0}\right)^{-1}$ are local real functions. The set $J_{r}(\boldsymbol{E}, 1)$ has a natural manifold structure; a divided chart $\left(x^{0}, x^{i}\right)$ on $E$ induce the chart $\left(x^{0}, x^{i}, x_{\underline{\alpha}}^{i}\right)$ on $J_{r}(E, 1)$ such that $x_{\underline{\alpha}}^{i} \circ s=\partial^{|\underline{\alpha}|} s^{i} / \partial x^{\underline{\alpha}}$ (here $\underline{\alpha}$ is a multiindex of length $|\underline{\alpha}|=r$ containing, in the 1-dimensional case, only the index 0 repeated $r$ times). The above charts yield a structure of a smooth manifold to $J_{k}(\boldsymbol{E}, 1)$. With respect to this smooth structure, the projections $\pi_{p}^{r}$ turn out to be smooth bundles, and in particular $\pi_{r}^{r+1}$ turn out to be affine bundles for $r \geq 1$. If $r=1$ then $J_{1}(\boldsymbol{E}, 1)=\operatorname{Proj}(\mathrm{T} \boldsymbol{E})$, the bundle of projective spaces obtained from each fibre of $T \boldsymbol{E} \rightarrow \boldsymbol{E}$.

Let $\boldsymbol{F}$ be another manifold such that $\operatorname{dim}(\boldsymbol{E}) \leq \operatorname{dim}(\boldsymbol{F})$. Let $f: \boldsymbol{E} \rightarrow \boldsymbol{F}$ be an immersion. Then $f$ maps (locally) 1-dimensional submanifolds of $\boldsymbol{E}$ to 1-dimensional submanifolds of $\boldsymbol{F}$ and preserves the contact, so we have the induced fibered morphisms $J_{r} f: J_{r}(\boldsymbol{E}, 1) \rightarrow J_{r}(\boldsymbol{F}, 1)$, over $f$. If we assume a vector field $X$ on $\boldsymbol{E}$, then its flow $F l_{t}^{X}$ is prolonged into the 1-parameter family of diffeomorphisms $J_{r}\left(F l_{t}^{X}\right): J_{r}(\boldsymbol{E}, 1) \rightarrow J_{r}(\boldsymbol{F}, 1)$ and we obtain the vector field $X_{(r)}:=\left.\frac{d}{d t}\right|_{0} J_{r}\left(F l_{t}^{X}\right)$, called the $r$-jet lift or the holonomic lift of vector fields on $\boldsymbol{E}$ to vector fields on $J_{r}(\boldsymbol{E}, 1)$. Let us denote by $\partial_{i}^{0}, \partial_{i}^{00}$, ... the coordinate vector fields with respect to the jet coordinates $x_{0}^{i}, x_{00}^{i}$, .... We have the coordinate expression

$$
\begin{aligned}
X_{(1)} & =X^{\lambda} \partial_{\lambda}+\left(\partial_{0} X^{i}+x_{0}^{p} \partial_{p} X^{i}-x_{0}^{i} \partial_{0} X^{0}-x_{0}^{i} x_{0}^{p} \partial_{p} X^{0}\right) \partial_{i}^{0} \\
& =X^{\lambda} \partial_{\lambda}+\breve{\delta}_{\lambda}^{i} \breve{\delta}_{0}^{\mu} \partial_{\mu} X^{\lambda} \partial_{i}^{0} .
\end{aligned}
$$

Here we use the notation $\breve{\delta}_{\lambda}^{i}=\delta_{\lambda}^{i}-x_{0}^{i} \delta_{\lambda}^{0}$ and $\breve{\delta}_{0}^{\mu}=\delta_{0}^{\mu}+x_{0}^{p} \delta_{p}^{\mu}$. We have $\left[X_{(1)}, \bar{X}_{(1)}\right]=[X, \bar{X}]_{(1)}$.

We introduce the bundle $T_{1,0} \boldsymbol{E}:=J_{1}(\boldsymbol{E}, 1) \times_{J_{r}(\boldsymbol{E}, 1)} T \boldsymbol{E}$; the pseudo-horizontal subbundle $H_{1,0} \boldsymbol{E} \subset T_{1,0} \boldsymbol{E}$ is defined by $H_{1,0} \boldsymbol{E}:=\left\{\left(j_{1} s(x), v\right) \in\right.$ 
$\left.T_{1,0} \boldsymbol{E} \mid v \in T_{j_{1} s(x)}\left(j_{1} s(\boldsymbol{S})\right)\right\}$. We also have the pseudo-vertical bundle $V_{1,0} \boldsymbol{E}:=$ $T_{1,0} \boldsymbol{E} / H_{1,0} \boldsymbol{E}$. The bundles $H_{1,0} \boldsymbol{E}$ and $V_{1,0} \boldsymbol{E}$ are strictly related with the horizontal and vertical bundle in the case of jets of fibrings. A local basis for the sections of the bundle $H_{1,0} \boldsymbol{E}$ is $D_{0}=\partial_{0}+x_{0}^{i} \partial_{i}$, while a local basis for the sections of its dual $H_{1,0}{ }^{*} \boldsymbol{E}$ is $\bar{d}^{0}:=\left.d^{0}\right|_{H_{1,0} \boldsymbol{E}}$. The fibred inclusion $D: J_{1}(\boldsymbol{E}, 1) \rightarrow H_{1,0}{ }^{*} \boldsymbol{E} \otimes T_{1,0} \boldsymbol{E}$ or, equivalently, the fibred projection $\omega: J_{1}(\boldsymbol{E}, 1) \rightarrow T_{1,0}{ }^{*} \boldsymbol{E} \otimes V_{1,0} \boldsymbol{E}$ is said to be the contact structure on $J_{1}(\boldsymbol{E}, 1)$. Their coordinate expressions are $D=\bar{d}^{0} \otimes D_{0}=\bar{d}^{0} \otimes\left(\partial_{0}+x_{0}^{i} \partial_{i}\right)$ and $\omega=\omega^{i} \otimes B_{i}$, where $\omega^{i}:=d^{i}-x_{0}^{i} d^{0}$, and $B_{i}=\left[\partial_{i}\right]$ is a local basis of $V_{1,0} \boldsymbol{E}$. Note that $\omega^{i}$ annihilate $D_{0}$; they are said to be contact forms, and generate a space which is naturally isomorphic to $V_{1,0}{ }^{*} \boldsymbol{E}$. Similar constructions can be repeated on $J_{r}(\boldsymbol{E}, 1)$ for any order $r$ (see [25] and references therein).

Lie derivatives can be used to characterize those vector fields on $J_{r}(\boldsymbol{E}, 1)$ that are the holonomic lift of a vector field on $\boldsymbol{E}$. For instance, a vector field $Y: J_{1}(\boldsymbol{E}, 1) \rightarrow T J_{1}(\boldsymbol{E}, 1)$ which projects onto a vector field $X$ on $T \boldsymbol{E}$ fulfills $Y=X_{(1)}$ if and only if $L_{(X, Y)} D=0$ or, equivalently, $L_{(X, Y)} D_{0}=f^{0} D_{0}$, where $f^{0}: J_{1}(\boldsymbol{E}, 1) \rightarrow \mathbb{R}$ is a smooth function.

A (second-order) differential equation is a submanifold $\mathcal{E} \subset J_{2}(\boldsymbol{E}, 1)$. A solution is a 1-dimensional submanifold $s$ of $\boldsymbol{E}$ such that $j_{2} s \subset \mathcal{E}$. We will describe the equation of particle motion in this way.

The calculus of variations can be formalized on jets of submanifolds ([5, 35]; see also [25]). Given a 1-form $\alpha$ on $J_{1}(\boldsymbol{E}, 1)$ and a 1-dimensional submanifold $s: \boldsymbol{L} \subset \boldsymbol{E}$ we have the action [25] $A_{\boldsymbol{U}}(s)=\int_{\boldsymbol{U}}\left(j_{1} s\right)^{*} \alpha$, where $\boldsymbol{U}$ is a regular oriented open 1-dimensional submanifold of $\boldsymbol{L}$ with compact closure. Contact forms do not contribute to the action. The horizontalization [25] takes the 1-form $\alpha$ into a section $h(\alpha)$ of $H_{1,0}^{*} \boldsymbol{E}$ which does not contain contact factors, hence $A_{\boldsymbol{U}}(s)=\int_{\boldsymbol{U}}\left(j_{2} s\right)^{*} h(\alpha)$. If $\alpha=\alpha_{0} d^{0}+\alpha_{i} d^{i}+\alpha_{i}^{0} d_{0}^{i}$ then we have the coordinate expression $h(\alpha)=h(\alpha)_{0} \bar{d}^{0}=\left(\alpha_{0}+\alpha_{i} x_{0}^{i}+\right.$ $\left.\alpha_{i}^{0} x_{00}^{i}\right) \bar{d}^{0}$. There exists a natural differential operator, the Euler-Lagrange operator $\mathcal{E}$, bringing a Lagrangian into its Euler-Lagrange expression. We have $\mathcal{E}(h(\alpha)): J_{3}(\boldsymbol{E}, 1) \rightarrow V_{1,0}^{*} \boldsymbol{E} \otimes H_{1,0}^{*} \boldsymbol{E}$. The corresponding Euler-Lagrange equations are

$$
\mathcal{E}(h(\alpha))=\left(\partial_{i} h(\alpha)_{0}-D_{0}\left(\partial_{i}^{0} h(\alpha)_{0}\right)+D_{00}\left(\partial_{i}^{00} h(\alpha)_{0}\right)\right) \omega^{i} \otimes \bar{d}^{0}=0,
$$

where $D_{00}=D_{0}^{2}$. The Euler-Lagrange equation can be achieved in another way, using properties of the $\mathcal{C}$-spectral sequence [5, 35]. Namely, it can be proved that $\mathcal{E}(h(\alpha))=h^{\prime}(d \alpha)$, where $h^{\prime}$ is horizontalization followed by factorization modulo total divergencies. 


\section{General relativistic mechanics}

In this section we summarize the model of general relativistic phase space by Janyška and Modugno (see [16] and references therein).

\subsection{Phase space.}

We assume the spacetime to be a manifold $\boldsymbol{E}$, with $\operatorname{dim} \boldsymbol{E}=4$, endowed with a scaled Lorentz metric $g: \boldsymbol{E} \rightarrow \mathbb{L}^{2} \otimes T^{*} \boldsymbol{E} \otimes_{\boldsymbol{E}} T^{*} \boldsymbol{E}$ whose signature is $(-+++)$. Moreover, we assume $\boldsymbol{E}$ to be oriented and time oriented. With reference to a mass $m \in \mathbb{M}$, it is convenient to introduce the rescaled metric $G:=\frac{m}{\hbar} g: \boldsymbol{E} \rightarrow \mathbb{T} \otimes\left(T^{*} \boldsymbol{E} \otimes_{\boldsymbol{E}} T^{*} \boldsymbol{E}\right)$. The associated contravariant tensors are $\bar{g}: \boldsymbol{E} \rightarrow \mathbb{L}^{-2} \otimes\left(T \boldsymbol{E} \otimes_{\boldsymbol{E}} T \boldsymbol{E}\right)$ and $\bar{G}=\frac{\hbar}{m} \bar{g}: \boldsymbol{E} \rightarrow \mathbb{T}^{*} \otimes\left(T \boldsymbol{E} \otimes_{\boldsymbol{E}} T \boldsymbol{E}\right)$. In what follows, we will not indicate obvious indexes in fibred (tensor) products.

A spacetime chart is defined to be a divided chart $\left(x^{0}, x^{i}\right)$ of $\boldsymbol{E}$ which fits the orientation of spacetime and such that the vector field $\partial_{0}$ is timelike and time oriented and the vector fields $\partial_{i}$ are spacelike. We have the coordinate expressions $g=g_{\varphi \psi} d^{\varphi} \otimes d^{\psi}, \bar{g}=g^{\varphi \psi} \partial_{\varphi} \otimes \partial_{\psi}$ where $g_{\varphi \psi}: E \rightarrow \mathbb{L}^{2} \otimes \mathbb{R}$ and $g^{\varphi \psi}: E \rightarrow \mathbb{L}^{-2} \otimes \mathbb{R}$ are mutually inverse matrices. Similarly $G=G_{\lambda \mu}^{0} u_{0} \otimes$ $d^{\lambda} \otimes d^{\mu}$ and $\bar{G}=G_{0}^{\lambda \mu} u^{0} \otimes \partial_{\lambda} \otimes \partial_{\mu}$ with $\left.G_{\lambda \mu}^{0}, G_{0}^{\lambda \mu}: \boldsymbol{E} \rightarrow \mathbb{R}\right)$.

A time-like 1-dimensional submanifold $s: \boldsymbol{T} \subset \boldsymbol{E}$ is said to be a motion, whose velocity is $j_{1} s$.

For every arbitrary choice of a proper time origin $t_{0} \in \boldsymbol{T}$, we obtain the proper time scaled function given by $\sigma: \boldsymbol{T} \rightarrow \overline{\mathbb{T}}: t \mapsto \frac{1}{c} \int_{\left[t_{0}, t\right]}\left\|\frac{d s}{d \breve{x}^{0}}\right\| d \breve{x}^{0}$. This map yields, at least locally, a bijection $\boldsymbol{T} \rightarrow \overline{\mathbb{T}}$, hence a (local) affine structure of $\boldsymbol{T}$ associated with the vector space $\overline{\mathbb{T}}$. Indeed, this (local) affine structure does not depend on the choice of the proper time origin and of the spacetime chart.

Let us choose a time origin $t_{0} \in \boldsymbol{T}$ and consider the associated proper time scaled function $\sigma: \boldsymbol{T} \rightarrow \overline{\mathbb{T}}$ and the induced linear isomorphism $T \boldsymbol{T} \rightarrow \boldsymbol{T} \times \overline{\mathbb{T}}$. Moreover, let us consider a spacetime chart $\left(x^{0}, x^{i}\right)$ and the induced chart $\left(\breve{x}^{0}\right)$ on $\boldsymbol{T}$. Let us set $\partial_{0} s^{i}:=\frac{d s^{i}}{d \breve{x}^{0}}$. The 1st differential of the motion $s$ is the map $d s:=\frac{d s}{d \sigma}: \boldsymbol{T} \rightarrow \mathbb{T}^{*} \otimes T \boldsymbol{E}$. We have $g(d s, d s)=-c^{2}$ and the coordinate expression

$$
g(d s, d s)=-c^{2}, \text { where } d s=\frac{c_{0} u^{0} \otimes\left(\left(\partial_{0} \circ s\right)+\partial_{0} s^{i}\left(\partial_{i} \circ s\right)\right)}{\sqrt{\left|\left(g_{00} \circ s\right)+2\left(g_{0 j} \circ s\right) \partial_{0} s^{j}+\left(g_{i j} \circ s\right) \partial_{0} s^{i} \partial_{0} s^{j}\right|}} .
$$

The open subbundle $\mathcal{J}_{1} \boldsymbol{E} \subset J_{1}(\boldsymbol{E}, 1)$ of velocities of motions is said to be the (general relativistic) phase space. In an analogous way we introduce the open subbundles $\partial_{r} \boldsymbol{E} \subset J_{r}(\boldsymbol{E}, 1)$. Any spacetime chart $\left(x^{0}, x^{i}\right)$ is adapted to 
each motion, hence the fibred chart $\left(x^{0}, x^{i} ; x_{0}^{i}\right)$ on $\mathcal{J}_{1} \boldsymbol{E}$ is global on the fibres of $\mathcal{\partial}_{1} \boldsymbol{E} \rightarrow \boldsymbol{E}$.

The restriction of the pseudo-horizontal bundle $H_{1,0} \boldsymbol{E}$ to $\mathcal{J}_{1} \boldsymbol{E}$ will be denoted by $\mathcal{H}_{1,0} \boldsymbol{E}$; it admits the trivialization $h_{g}: \mathcal{H}_{1,0} \boldsymbol{E} \rightarrow \mathcal{J}_{1} \boldsymbol{E} \times \overline{\mathbb{T}},\left(j_{1} s(x), v\right) \rightarrow$ $\left(j_{1} s(x), \pm \frac{\|v\|}{c}\right)$, where the sign depends on the time orientation of $v$. Simple computations show that the scaled vector field

$$
\text { д }:=\left.\left(\left(h_{g}{ }^{-1}\right)^{*} \otimes \operatorname{id}_{T \boldsymbol{E}}\right) \circ D\right|_{\partial_{1} \boldsymbol{E}}: \mathcal{\partial}_{1} \boldsymbol{E} \rightarrow \mathbb{T}^{*} \otimes T \boldsymbol{E}
$$

has coordinate expression д $=c \alpha^{0} D_{0}$, where $\alpha^{0}:=1 /\|D\|_{0}=\mid g_{00}+2 g_{0 j} x_{0}^{j}+$ $\left.g_{i j} x_{0}^{i} x_{0}^{j}\right|^{-1 / 2}$. The vector field д is said to be the normalized contact structure. It is characterised by the equality д $о j_{1} s=d s$ for every motion $s$. We have $g \circ($ д, д $)=-c^{2}$, hence $\mathcal{J}_{1} \boldsymbol{E}$ can be regarded as a non-linear subbundle $\mathcal{J}_{1} \boldsymbol{E} \subset \mathbb{T}^{*} \otimes T \boldsymbol{E}$ whose fibres are diffeomorphic to $\mathbb{R}^{3}$.

2.1 Remark. The property (11) says that $\mathcal{J}_{1} \boldsymbol{E}$ is a universal mass shell, i.e. it represents a mass shell for each choice of particle mass $m \in \mathbb{M}$. At the same time, (1) says that $\partial_{1} \boldsymbol{E}$ coincides with the pseudosphere bundle in [11]. The advantage of the jet space picture is that we can use natural jet coordinates instead of dealing with a constraint, and at the same time we give a geometric interpretation of projective coordinates used by other authors [28]

We also have the dual counterpart of д

$$
\tau:=-c^{-2} g^{\mathrm{b}} \text { ○ д: } \mathcal{J}_{1} \boldsymbol{E} \rightarrow \mathbb{T} \otimes T^{*} \boldsymbol{E},
$$

with coordinate expression $\tau=\tau_{\lambda} d^{\lambda}=-c^{-1} \alpha^{0}\left(g_{0 \lambda}+g_{i \lambda} x_{0}^{i}\right) d^{\lambda}$.

The metric $g$ yields an orthogonal splitting of the tangent space $T \boldsymbol{E}$ on each $x \in \boldsymbol{E}$ on which a time-like direction has been assigned. Hence, we have the splitting 14, 16.

$$
\mathcal{T}_{1,0} \boldsymbol{E}=\mathcal{H}_{1,0} \underset{\partial_{1} \boldsymbol{E}}{\oplus} \mathcal{V}_{g} \boldsymbol{E}
$$

where $\mathcal{T}_{1,0} \boldsymbol{E}=\left.T_{1,0} \boldsymbol{E}\right|_{\mathfrak{f}_{1} \boldsymbol{E}}$. Let us introduce the notation $\mathcal{V}_{1,0} \boldsymbol{E}:=\left.V_{1,0} \boldsymbol{E}\right|_{\mathfrak{f}_{1} \boldsymbol{E}}$. The projection on $\mathcal{V}_{g} \boldsymbol{E}$ is denoted by $\theta$; it is readily proved that $\theta=$ $\operatorname{id}_{\mathcal{T}_{1,0} \boldsymbol{E}}-\tau \otimes$ д. We will use the local basis $\left(D_{0}, N_{i}\right)$ adapted to the splitting (44), where $N_{i}:=\partial_{i}-c \alpha^{0} \tau_{i} D_{0}$ is the basis of $\mathcal{V}_{g} \boldsymbol{E}$. Note that $\left.\omega\right|_{\mathcal{T}_{1,0}}$ restricts to $\mathcal{V}_{g} \boldsymbol{E}$ to an isomorphism $\mathcal{V}_{g} \boldsymbol{E} \rightarrow \mathcal{V}_{1,0} \boldsymbol{E}$; it reads in coordinates as $N_{i} \rightarrow B_{i}$. The dual splitting of (4) has a local basis dual to $\left(D_{0}, N_{i}\right)$, namely $\left(N^{0}, \omega^{i}\right)$, where $N^{0}=d^{0}+c \alpha^{0} \tau_{i} \omega^{i}$ and $\omega^{i}$ are contact forms (see the Preliminaries). 
In what follows it is convenient to set $\breve{g}_{0 \lambda}:=g\left(D_{0}, \partial_{\lambda}\right)=\breve{\delta}_{0}^{\rho} g_{\rho \lambda}, \hat{g}_{00}=$ $g\left(D_{0}, D_{0}\right)=\breve{\delta}_{0}^{\rho} \breve{\delta}_{0}^{\sigma} g_{\rho \sigma}=-\left(\alpha^{0}\right)^{-2}, \breve{g}^{i \lambda}=\bar{g}\left(\omega^{i}, d^{\lambda}\right)=\breve{\delta}_{\sigma}^{i} g^{\sigma \lambda}$. We will use a similar notation for the rescaled metric $G$.

According to the above splitting we have the decomposition $g \circ \pi_{0}^{1}=g_{\|}+g_{\perp}$ of the lift of $g$ on $\mathcal{T}_{1,0} \boldsymbol{E}$, where $g_{\|}$is a negative-definite metric on $\mathcal{H}_{1,0}$ and $g_{\perp}$ is a Riemannian metric on $\mathcal{V}_{g} \boldsymbol{E}$. The coordinate expression of the above metric and of their contravariant form $\bar{g}_{\|}$and $\bar{g}_{\perp}$ with respect to the local basis $\left(D_{0}, N_{i}\right)$ and its dual $\left(N^{0}, \omega^{i}\right)$ are

$$
\begin{array}{ll}
g_{\| 00}=\left(\alpha^{0}\right)^{-2}, & \bar{g}_{\|}^{00}=\left(\alpha^{0}\right)^{2}, \\
g_{\perp i j}=g_{i j}+c^{2} \tau_{i} \tau_{j}, & \bar{g}_{\perp}^{i j}=g^{i j}-g^{i 0} x_{0}^{j}-g^{0 j} x_{0}^{i}+g^{00} x_{0}^{i} x_{0}^{j} .
\end{array}
$$

Note that the trivialization $h_{g}$ together with the splitting (44) yields the further splitting $\mathcal{T}_{1,0} \boldsymbol{E}=\overline{\mathbb{T}} \underset{\jmath_{1} \boldsymbol{E}}{\oplus} \mathcal{V}_{g} \boldsymbol{E}$; here the expressions of $g_{\|}$and $\bar{g}_{\|}$are $g_{\| 00}=c^{2}$ and $\bar{g}_{\|}^{00}=c^{-2}$.

The vertical derivative $V$ д induces the linear fibred isomorphisms

$$
v_{g}: V \mathcal{J}_{1} \boldsymbol{E} \rightarrow \mathbb{T}^{*} \otimes \mathcal{V}_{g} \boldsymbol{E}, \quad v_{g}^{-1}: \mathbb{T}^{*} \otimes \mathcal{V}_{g} \boldsymbol{E} \rightarrow V \mathcal{J}_{1} \boldsymbol{E}
$$

over $\partial_{1} \boldsymbol{E}$, with coordinate expressions $v_{g}=c \alpha^{0} d_{0}^{i} \otimes N_{i}$ and $v_{g}^{-1}=\frac{1}{c \alpha^{0}} \omega^{i} \otimes \partial_{i}^{0}$.

We define a spacetime connection to be a torsion free linear connection $K: T \boldsymbol{E} \rightarrow T^{*} \boldsymbol{E} \otimes T T \boldsymbol{E}$ of the bundle $T \boldsymbol{E} \rightarrow \boldsymbol{E}$. Its coordinate expression is of the type

$$
K=d^{\lambda} \otimes\left(\partial_{\lambda}+K_{\lambda}{ }^{\nu}{ }_{\mu} \dot{x}^{\mu} \dot{\partial}_{\nu}\right), \quad \text { with } \quad K_{\mu}{ }^{\nu}{ }_{\lambda}=K_{\lambda}{ }^{\nu}{ }_{\mu}: \boldsymbol{E} \rightarrow \mathbb{R} .
$$

We define a phase connection to be a connection of the bundle $\mathcal{\partial}_{1} \boldsymbol{E} \rightarrow \boldsymbol{E}$. A phase connection can be represented, equivalently, by $\Gamma: \mathcal{J}_{1} \boldsymbol{E} \rightarrow T^{*} \boldsymbol{E} \otimes$ $T \mathcal{J}_{1} \boldsymbol{E}$, which is projectable over $\mathrm{id}_{T \boldsymbol{E}}$, or by $\nu[\Gamma]=\operatorname{id}_{T \mathfrak{d}_{1} \boldsymbol{E}}-\Gamma: \mathcal{J}_{1} \boldsymbol{E} \rightarrow$ $T^{*} \mathcal{J}_{1} \boldsymbol{E} \otimes V \mathcal{J}_{1} \boldsymbol{E}$, or $v_{g}[\Gamma]:=v_{g} \circ \nu[\Gamma]: \mathcal{J}_{1} \boldsymbol{E} \rightarrow T^{*} \mathcal{J}_{1} \boldsymbol{E} \otimes\left(\mathbb{T}^{*} \otimes \mathcal{V}_{g} \boldsymbol{E}\right)$. Their coordinate expressions are

$$
\begin{aligned}
\Gamma & =d^{\lambda} \otimes\left(\partial_{\lambda}+\Gamma_{\lambda_{0}}^{i} \partial_{i}^{0}\right), & \nu[\Gamma]=\left(d_{0}^{i}-\Gamma_{\lambda_{0}}^{i} d^{\lambda}\right) \otimes \partial_{i}^{0}, \\
v_{g}[\Gamma] & =c \alpha^{0}\left(d_{0}^{i}-\Gamma_{\lambda_{0}}^{i} d^{\lambda}\right) \otimes N_{i}, & \text { with } \quad \Gamma_{\lambda_{0}}^{i}: \mathcal{\partial}_{1} \boldsymbol{E} \rightarrow \mathbb{R} .
\end{aligned}
$$

A spacetime connection $K$ induces naturally a phase connection $\Gamma=$ $\chi(K)$ [16], which is expressed in coordinates by $\Gamma_{\varphi 0}^{i}=\breve{\delta}_{\sigma}^{i} K_{\varphi}{ }^{\sigma}{ }_{\rho} \breve{\delta}_{0}^{\rho}$.

We denote by $K^{\mathfrak{g}}$ the Levi Civita connection, i.e. the torsion free linear spacetime connection such that $\nabla g=0$. The connections $K^{\mathfrak{g}}$ and $\Gamma^{\mathfrak{g}}:=\chi\left(K^{\mathfrak{g}}\right)$ are said to be gravitational. 


\subsection{Gravitational and electromagnetic forms.}

Of course, being $\operatorname{dim} \mathcal{J}_{1} \boldsymbol{E}=7$, there exist no symplectic forms on $\mathcal{J}_{1} \boldsymbol{E}$. Hence, we are going to define a contact structure (in the sense of [22]) on $\mathcal{J}_{1} \boldsymbol{E}$. The gravitational connection $\Gamma^{\mathfrak{g}}$ and the rescaled metric $G$ induce the 2 -form on $\partial_{1} \boldsymbol{E}$

$$
\left.\Omega^{\mathfrak{g}}:=G_{\perp}\right\lrcorner\left(v_{g}\left[\Gamma^{\mathfrak{g}}\right]\right) \wedge \theta: \mathcal{J}_{1} \boldsymbol{E} \rightarrow \bigwedge^{2} T^{*} \mathcal{J}_{1} \boldsymbol{E} .
$$

It can be proved [16] that $\Omega^{\mathfrak{g}}=-\frac{m c^{2}}{\hbar} d \tau$, hence $\Omega^{\mathfrak{g}}$ is an exact form. Moreover, the form $\tau \wedge \Omega^{\mathfrak{g}} \wedge \Omega^{\mathfrak{g}} \wedge \Omega^{\mathfrak{g}}$ is a scaled volume form on $\mathcal{J}_{1} \boldsymbol{E}$, hence $\Omega^{\mathfrak{g}}$ is non degenerate. It turns out that the pair $\left(-\widehat{\tau}:=-\frac{m c^{2}}{\hbar} \tau, \Omega^{\mathfrak{g}}\right)$ define on $\partial_{1} \boldsymbol{E}$ a contact structure. $\Omega^{\mathfrak{g}}$ is said to be the phase gravitational 2-form of our model. We have the coordinate expression

$$
\Omega^{\mathfrak{g}}=c_{0} \alpha^{0} G_{\perp i j}^{0}\left(d_{0}^{i}-\Gamma_{\varphi 0}^{\mathfrak{g}} \underset{\varphi}{i} d^{\varphi}\right) \wedge \omega^{j} .
$$

Now, we assume the electromagnetic field to be a closed scaled 2-form on $E$

$$
F: \boldsymbol{E} \rightarrow\left(\mathbb{L}^{1 / 2} \otimes \mathbb{M}^{1 / 2}\right) \otimes \bigwedge^{2} T^{*} \boldsymbol{E}
$$

We have the coordinate expression $F=2 F_{0 j} d^{0} \wedge d^{j}+F_{i j} d^{i} \wedge d^{j}$. We denote a local potential of $F$ with $A: \boldsymbol{E} \rightarrow T^{*} \boldsymbol{E}$, according to $2 d A=F$. In what follows we shall use the unscaled electromagnetic 2 -form $\widehat{F}:=(q / \hbar) F$ and its potential $\widehat{A}:=(q / \hbar) A$.

Given a charge $q$, the rescaled electromagnetic field $(q / 2 \hbar) F$ can be incorporated into the geometrical structure of the phase space, i.e. the gravitational form. Namely, we define the joined (total) phase 2-form

$$
\Omega:=\Omega^{\mathfrak{g}}+\frac{q}{2 \hbar} F: \partial_{1} \boldsymbol{E} \rightarrow \bigwedge^{2} T^{*} \mathcal{J}_{1} \boldsymbol{E} .
$$

Of course $d \Omega=0$ but $\Omega$ is exact if and only if $F$ is exact. A (locally defined) 1 -form $\Theta$ such that $\Omega=d \Theta$ is said to be a potential of $\Omega$. Every potential of $\Omega$ can be written as $\Theta=-\widehat{\tau}+\widehat{A}$ up to a closed form. A potential $\Theta$ is locally defined because the potential $A$ is locally defined. We have $\tau \wedge \Omega \wedge \Omega \wedge \Omega=\tau \wedge \Omega^{\mathfrak{g}} \wedge \Omega^{\mathfrak{g}} \wedge \Omega^{\mathfrak{g}}$, so $\Omega$ is non degenerate. Hence, the pair $(-\widehat{\tau}, \Omega)$ is an almost-cosymplectic-contact (in the sense of [17]) encoding the gravitational and electromagnetic (classical) structure of the phase space. Note that, even locally, $(-\widehat{\tau}+\widehat{A}, \Omega)$ cannot be regarded as a contact pair, because the potential of $\Omega$ could vanish at some point. 
We recall that a unique connection $\Gamma$ on $\mathcal{J}_{1} \boldsymbol{E} \rightarrow \boldsymbol{E}$ can be characterized through the total 2 -form $\Omega$ [16] by the formula (66). Namely the joined (total) phase connection $\Gamma=\Gamma^{\mathfrak{g}}+\Gamma^{\mathfrak{e}}$, where

$$
\left.\Gamma^{\mathfrak{e}}:=-\frac{1}{2} v_{g}^{-1} \circ G^{\sharp 2} \circ(\widehat{F}+2 \tau \wedge(\text { д }\lrcorner \widehat{F})\right)
$$

with the coordinate expression $\Gamma^{\mathfrak{e}}=-\left(1 /\left(2 c_{0} \alpha^{0}\right)\right) \breve{G}_{0}^{i \mu}\left(\widehat{F}_{\lambda \mu}-\left(\alpha^{0}\right)^{2} \breve{g}_{0 \lambda} \widehat{F}_{\rho \mu} \breve{\delta}_{0}^{\rho}\right) d^{\lambda} \otimes$ $\partial_{i}^{0}$.

\subsection{Equation of particle motion.}

We can define a second order contact structure д2 $_{2}: \mathcal{J}_{2} \boldsymbol{E} \rightarrow \mathbb{T}^{*} \otimes_{\mathfrak{J}_{2} \boldsymbol{E}}^{\otimes} T \mathcal{J}_{1} \boldsymbol{E}$. Then, the map д $\lrcorner \Gamma$ takes its values in the subbundle $\mathcal{J}_{2} \boldsymbol{E}$.

We define a second order dynamical connection $\gamma[16]$ on spacetime as the map

$$
\gamma:=\text { д }\lrcorner \Gamma: \mathcal{\partial}_{1} \boldsymbol{E} \rightarrow \mathcal{\partial}_{2} \boldsymbol{E} \stackrel{\text { Д2 }_{2}}{\hookrightarrow} \mathbb{T}^{*} \otimes T \mathcal{J}_{1} \boldsymbol{E} .
$$

The above map $\gamma$ plays here a role analogous to that of the geodesic spray in the formulation of Galilean mechanics on tangent spaces. We have the coordinate expressions

$$
\gamma=c \alpha^{0}\left(\partial_{0}+x_{0}^{i} \partial_{i}+\gamma_{00}^{i} \partial_{i}^{0}\right), \quad \gamma_{00}^{i}:=x_{00}^{i} \circ \gamma=\Gamma_{00}^{i}+\Gamma_{j 0}^{i} x_{0}^{j} .
$$

For the gravitational phase connection we have the gravitational second order connection $\gamma^{\mathfrak{g}}:=$ д $\lrcorner \Gamma^{\mathfrak{g}}$. As a scaled vector field on $\mathcal{J}_{1} \boldsymbol{E}$, $\gamma^{\mathfrak{g}}$ fulfills $\left.\left.\gamma^{\mathfrak{g}}\right\lrcorner \Omega^{\mathfrak{g}}=0, \gamma^{\mathfrak{g}}\right\lrcorner \tau=1$, hence $\widehat{\gamma}^{\mathfrak{g}}:=\frac{\hbar}{m c^{2}} \gamma^{\mathfrak{g}}$ is the Reeb vector field associated with the contact pair $\left(-\widehat{\tau}, \Omega^{\mathfrak{g}}\right)[22]$. The dual Jacobi structure is given by the pair $\left(-\widehat{\gamma}^{\mathfrak{g}}, \Lambda^{\mathfrak{g}}\right)$, where $\Lambda^{\mathfrak{g}}$ is the gravitational phase 2 -vector given by

$$
\left.\Lambda^{\mathfrak{g}}:=\bar{G}\right\lrcorner\left(\Gamma^{\mathfrak{g}} \wedge v_{g}^{-1}\right): \mathcal{J}_{1} \boldsymbol{E} \rightarrow \bigwedge^{2} T \mathcal{J}_{1} \boldsymbol{E}
$$

with coordinate expression $\Lambda^{\mathfrak{g}}=\left(1 /\left(c_{0} \alpha^{0}\right)\right) \breve{G}_{0}^{j \lambda}\left(\partial_{\lambda}+\Gamma_{\lambda_{0}}^{i} \partial_{i}^{0}\right) \wedge \partial_{j}^{0}$.

It is natural to ask about analogous properties of the total pair $(-\widehat{\tau}, \Omega)$. It can be easily proved that there exists a second order connection $\gamma$ such that $\gamma\lrcorner \Omega=0$ and $\gamma\lrcorner \tau=1$, i.e. $\widehat{\gamma}:=\frac{\hbar}{m c^{2}} \gamma$ is the Reeb vector field associated with the almost-cosymplectic-contact pair $(-\widehat{\tau}, \Omega)$ [17]. Such a second order connection takes the form $\gamma=\gamma^{\mathfrak{g}}+\gamma^{e}$, where

$$
\gamma^{e}: \mathcal{J}_{1} \boldsymbol{E} \rightarrow \mathbb{T}^{*} \otimes V \mathcal{J}_{1} \boldsymbol{E}
$$


Note that the above sum is performed in $\mathcal{J}_{2} \boldsymbol{E}$, as $\mathbb{T}^{*} \otimes V \mathcal{J}_{1} \boldsymbol{E}$ is the associated fibre bundle [25] to the affine bundle $\pi_{1}^{2}: \mathcal{J}_{2} \boldsymbol{E} \rightarrow \mathcal{J}_{1} \boldsymbol{E}$. We have the coordinate expression

$$
\gamma^{e}=-\breve{G}_{0}^{i \lambda}\left(\widehat{F}_{0 \lambda}+\widehat{F}_{j \lambda} x_{0}^{j}\right) u^{0} \otimes \partial_{i}^{0} .
$$

Of course, $\gamma^{e}$ is the Lorentz force associated with $F$.

The equation of particle motion is the submanifold of $\partial_{2} \boldsymbol{E}$ defined as follows

$$
\nabla[\gamma]=j_{2} s-\gamma \circ j_{1} s=0 .
$$

In other words, the equation of motion is the image of the section $\gamma$. It is equivalent to $j_{2} s-\gamma^{\mathfrak{g}} \circ j_{1} s=\gamma^{e} \circ j_{1} s$. We have the coordinate expression

$$
x_{00}^{i}-\breve{\delta}_{\tau}^{i} K_{\rho \sigma}^{\tau} \breve{\delta}_{0}^{\rho} \breve{\delta}_{0}^{\sigma}=-\frac{q}{m} \bar{g}_{\perp}^{i k} \breve{\delta}_{0}^{\rho} F_{\rho k} .
$$

2.2 Remark. The above system of equations is different from the usual equations of general relativistic mechanics. The main difference is that the above equations are on unparametrized trajectories rather than on parametrized trajectories. Indeed, it is well-known that general relativistic mechanics is invariant with respect to reparametrizations [8, 10]. The above approach allows us to discard the extra degree of freedom constituted by the parameter of motion. Hence, the equations of motion are just 3 instead of the standard 4 , at the cost of having a polynomial expression of degree 3 in velocities $x_{0}^{i}$ instead of 2 , like in the standard parametrized geodesic equation. Mathematically, this corresponds to the fact that it is possible to regard $J_{1}(\boldsymbol{E}, 1)$ as the quotient $\operatorname{reg} T_{1}^{1} \boldsymbol{E} / G_{1}^{1}$, where $\operatorname{reg} T_{1}^{1} \boldsymbol{E}$ is the space of regular 1-velocities of curves (here it coincides with the tangent bundle to $\boldsymbol{E}$ without the zero section) and $G_{1}^{1}$ is the 1-jet of reparametrizations of curves.

The dual almost-coPoisson-Jacobi structure associated with the almostcosymplectic-contact structure $(-\widehat{\tau}, \Omega)$ is the pair $(-\widehat{\gamma}, \Lambda)$, where the joined (total) phase 2-vector $\Lambda:=\bar{G}\lrcorner\left(\Gamma \wedge v_{g}^{-1}\right)$, splits as $\Lambda=\Lambda^{\mathfrak{g}}+\Lambda^{\mathfrak{e}}$, where

$$
\Lambda^{\mathfrak{e}}=\frac{1}{2}\left(v_{g}^{-1} \wedge v_{g}^{-1}\right)\left(G^{\sharp}\left(\theta^{*}(\widehat{F})\right)\right),
$$

i.e., in coordinates, $\Lambda^{\mathfrak{e}}=\left(1 /\left(2\left(c_{0} \alpha^{0}\right)^{2}\right)\right) \breve{G}_{0}^{i \lambda} \breve{G}_{0}^{j \mu} \widehat{F}_{\lambda \mu} \partial_{i}^{0} \wedge \partial_{j}^{0}$.

Let us remark that if we consider the unscaled 1-form $\widehat{\tau}=\frac{m c^{2}}{\hbar} \tau$ and the unscaled vector field $\widehat{\gamma}=\frac{\hbar}{m c^{2}} \gamma$ we can split the tangent and the cotangent bundles of the phase space as

$$
\begin{aligned}
T \partial_{1} \boldsymbol{E} & =H_{\gamma} \partial_{1} \boldsymbol{E} \oplus V_{\tau} \mathcal{\partial}_{1} \boldsymbol{E}=\langle\widehat{\gamma}\rangle \oplus \operatorname{ker}(\widehat{\tau}), \\
T^{*} \mathcal{J}_{1} \boldsymbol{E} & =H_{\tau}^{*} \mathcal{J}_{1} \boldsymbol{E} \oplus V_{\gamma}^{*} \mathcal{J}_{1} \boldsymbol{E}=\langle\widehat{\tau}\rangle \oplus \operatorname{ker}(\widehat{\gamma}),
\end{aligned}
$$


where $H_{\gamma} \mathcal{\partial}_{1} \boldsymbol{E}:=\langle\widehat{\gamma}\rangle, V_{\gamma}^{*} \mathcal{J}_{1} \boldsymbol{E}:=\operatorname{ker}(\widehat{\gamma}), H_{\tau}^{*} \mathcal{J}_{1} \boldsymbol{E}:=\langle\widehat{\tau}\rangle, V_{\tau} \mathcal{\partial}_{1} \boldsymbol{E}:=\operatorname{ker}(\widehat{\tau})$. We have the mutually inverse isomorphisms $\Lambda^{\sharp}: V_{\gamma}^{*} \mathcal{J}_{1} \boldsymbol{E} \rightarrow V_{\tau} \mathcal{J}_{1} \boldsymbol{E}$ and $\Omega^{b}$ : $V_{\tau} \mathcal{\partial}_{1} \boldsymbol{E} \rightarrow V_{\gamma}^{*} \mathcal{\partial}_{1} \boldsymbol{E}$

The equation of motion can be derived from the variational viewpoint, see 24; here we recall the main steps. Taking the horizontal part of $\Omega$ we have $h(\Omega)=\eta$, where $\eta: \mathcal{J}_{2} \boldsymbol{E} \rightarrow \mathcal{V}_{g}^{*} \boldsymbol{E}{\otimes \mathfrak{f}_{2} \boldsymbol{E}}_{\mathcal{H}_{1,0}^{*}} \boldsymbol{E}$ has the coordinate expression

$$
\eta=\frac{m}{\hbar}\left(c \alpha^{0} g_{\perp i j}\left(x_{00}^{i}-\gamma^{\mathfrak{g}}{ }_{00}^{i}\right)-\frac{q}{m}\left(F_{0 j}+F_{i j} x_{0}^{i}\right)\right) \omega^{j} \otimes \bar{d}^{0} .
$$

Indeed, the form $\Omega$ can be locally split as $\Omega=\eta+C$, where $C$ is a contact form. We observe that $\eta$ is a Euler-Lagrange morphism: it is divergencefree because it takes values in a space of divergence-free forms [25]. Now, the obvious link between $\eta$ and the equation of motion is $\eta=\bar{g}_{\perp}(\nabla[\gamma])$. Of course, $\eta$ comes from a Lagrangian: the relation $\Omega=d \Theta=d(-\widehat{\tau}+\widehat{A})$ implies that $\eta$ admits the (local) Lagrangian $\mathcal{L}: \mathcal{J}_{1} \boldsymbol{E} \rightarrow \mathcal{H}_{1,0}^{*} \boldsymbol{E}, \mathcal{L}=h(\Theta)$, with coordinate expression

$$
\mathcal{L}=L_{0} \bar{d}^{0}=\left(-\frac{m c}{\hbar \alpha^{0}}+\left(\widehat{A}_{0}+x_{0}^{i} \widehat{A}_{i}\right)\right) \bar{d}^{0} .
$$

Note that the condition $-\frac{m c}{\hbar \alpha^{0}}+\left(\widehat{A}_{0}+x_{0}^{i} \widehat{A}_{i}\right) \neq 0$ is equivalent to the fact that $(-\widehat{\tau}+\widehat{A}) \wedge \Omega^{3}$ is a volume form, and in this case $(\Theta, \Omega)$ is a contact structure on the region of the phase space where $A$ is defined and $\Theta \neq 0$. Of course, $\mathcal{L}$ is defined on the same domain as $A$, and it is global if and only if $F$ is exact (including the distinguished case $F=0$ ). A simple computation proves that $\Theta$ is the Poincaré-Cartan form associated with the Lagrangian of the above theorem.

The Hessian of $\mathcal{L}$ is a non-singular matrix. In particular we have $\frac{\partial^{2} L}{\partial x_{0}^{i} \partial x_{0}^{j}}=$ $-\frac{m c}{\hbar} \alpha^{0} g_{\perp i j}$. Note that in the standard relativistic mechanics the arc-length Lagrangian $\tilde{\mathcal{L}}=\sqrt{g_{A B} \dot{y}^{A} \dot{y}^{B}}$ on $T \boldsymbol{E}$ has an Hessian matrix whose rank is 3, hence it is singular. Indeed, we can regard $д_{1}: \mathcal{\partial}_{1} \boldsymbol{E} \rightarrow \mathbb{T}^{*} \otimes T \boldsymbol{E}$ as a universal model of all primary constraints which are characterized by 1 . Such primary constraints are called mass shells, and are uniquely determined by the choice of a time scale $u_{0} \in \mathbb{T}$.

The Hamiltonian formalism is observer-dependent, so we introduce observers in our setting. A section $o: \boldsymbol{E} \rightarrow \mathcal{J}_{1} \boldsymbol{E}$ is said to be an observer; $o$ can also be interpreted as a vector-valued form $o=\bar{d}^{0} \otimes\left(\partial_{0}+o_{0}^{i} \partial_{i}\right)$, where $o_{0}^{i}=x_{0}^{i} \circ o$. An integral motion of an observer is a motion $s$ such that $j_{1} s=o \circ s$. There exists spacetime coordinates for which $o_{0}^{i}=0$; such coordinates are said to be adapted to $o$. Any observer $o: \boldsymbol{E} \rightarrow \mathcal{J}_{1} \boldsymbol{E}$ yields a projection of $T^{*} \boldsymbol{E}$-valued forms onto $\mathcal{H}_{1,0} \boldsymbol{E}$. The observed Hamiltonian $\mathcal{H}[o]$ 
is defined to be the projection of the Poincaré-Cartan form $\Theta$ onto $\mathcal{H}_{1,0} \boldsymbol{E}$ through $o$ :

$$
\mathcal{H}[o]=-o\lrcorner \Theta=-\left(\frac{m c}{\hbar} \alpha^{0}\left(g_{00}+g_{i 0} x_{0}^{i}\right)+\widehat{A}_{0}\right) \bar{d}^{0} .
$$

The observed Hamiltonian and the (local) Lagrangian are connected by the Legendre transformation as follows. Consider the momentum $\mathcal{P}:=V_{\boldsymbol{E}} \mathcal{L}: \mathcal{J}_{1} \boldsymbol{E}$ $\rightarrow V_{\boldsymbol{E}}^{*} \mathcal{J}_{1} \boldsymbol{E} \otimes \mathcal{H}_{1,0} \boldsymbol{E} \simeq \mathcal{V}_{1,0}^{*} \boldsymbol{E}$. Then, by means of the inclusion $\omega: \mathcal{V}_{1,0}^{*} \boldsymbol{E} \rightarrow$ $\mathcal{T}_{1,0}^{*} \boldsymbol{E}$ and the observer we can introduce the observed momentum $\mathcal{P}[o]:=(\omega \circ$ $o)\lrcorner \mathcal{P}$, with coordinate expression (with respect to coordinates adapted to $o$ ) $\mathcal{P}[o]=\partial_{i}^{0} L_{0} d^{i}$. Then a simple computation shows that the following identity holds:

$$
\mathcal{H}[o]=h(\mathcal{P}[o])-\mathcal{L}, \quad h(\mathcal{P}[o])=\partial_{i}^{0} L_{0} x_{0}^{i} \bar{d}^{0}
$$

\subsection{Examples}

In this section we consider two simple general relativistic spacetimes, Minkowski and Reissner-Nordstrom, and compute coordinate expressions for the geometric objects of our model.

Minkowski spacetime. We assume $\boldsymbol{E}=\mathbb{R}^{4}$ and $g=l_{0}^{2} \otimes g^{0}$, where $l_{0}^{2}$ is a unit of measurement of area and $g^{0}$ is the usual Minkowski metric; we also assume $F=0$. We have the Cartesian coordinate expressions:

$$
\begin{aligned}
& \alpha^{0}=\left(\sqrt{\left|-1+\left(x_{0}^{1}\right)^{2}+\left(x_{0}^{2}\right)^{2}+\left(x_{0}^{3}\right)^{2}\right|}\right)^{-1}, \\
& \tau=-c^{-1} \alpha^{0}\left(-d^{0}+x_{0}^{1} d^{1}+x_{0}^{2} d^{2}+x_{0}^{3} d^{3}\right) .
\end{aligned}
$$

Note that all Christoffel symbols $K$ and $\Gamma$ are zero. Being $G_{\perp i j}=\frac{m}{\hbar}\left(\delta_{i j}+\right.$ $\left.\left(\alpha^{0}\right)^{2} x_{0}^{i} x_{0}^{j}\right)$, it follows that

$$
\Omega=c_{0} \alpha^{0} \frac{m}{\hbar} \sum_{i, j=1}^{3}\left(\delta_{i j}+\left(\alpha^{0}\right)^{2} x_{0}^{i} x_{0}^{j}\right) d_{0}^{i} \wedge \omega^{j},
$$

Note that in this case $\Theta=-\tau$ and $\gamma=$ д. Of course, Euler-Lagrange equations reduce to $x_{00}^{i}=0$ even if the Lagrangian is $L_{0}=-(m c) /\left(\hbar \alpha^{0}\right)$. Since $M$ is parallelizable, its jet space is also trivial: $J_{1}(\boldsymbol{E}, 1)=\boldsymbol{E} \times \mathbb{P R}^{4}$ and $\mathcal{J}_{1} \boldsymbol{E}$ can be identified with the subspace $\boldsymbol{E} \times \mathbb{R}^{3}$ of time-like vectors. It follows that we can choose the observer $o=0$ induced by the canonical basis of $\mathbb{R}^{4}$. With respect to such an observer, the Hamiltonian takes the form $\mathcal{H}[o]=-(m c / \hbar) \alpha^{0} \bar{d}^{0}$. 
Reissner-Nordstrom spacetime. We assume $\boldsymbol{E}=\mathbb{R} \times\left(\mathbb{R}^{3} \backslash\{0\}\right)$ with local coordinates $\left(x^{0}, x^{1}, x^{2}, x^{3}\right)=(t, r, \theta, \varphi)$. We also assume a metric $g$ and an electromagnetic field $\widehat{A}$, where $g=l_{0}^{2} \otimes g^{0}, l_{0}^{2}$ is a unit of measurement of area and

$$
\begin{aligned}
g^{0}= & g_{00} d t \otimes d t+g_{11} d r \otimes d r+g_{22} d \theta \otimes d \theta+g_{33} d \varphi \otimes d \varphi \\
= & -\left(1-\frac{k_{s}}{r}+\frac{k_{q}^{2}}{r^{2}}\right) d t \otimes d t+\left(1-\frac{k_{s}}{r}+\frac{k_{q}^{2}}{r^{2}}\right)^{-1} d r \otimes d r \\
& +r^{2}\left(d \theta \otimes d \theta+\sin ^{2} \theta d \varphi \otimes d \varphi\right) . \\
\widehat{A}= & -\left(q_{0} /\left(\hbar_{0} r\right)\right) d^{0} .
\end{aligned}
$$

Here coordinates on velocities are denoted by $r_{0}, \theta_{0}, \varphi_{0}$, the constant $k_{s}$ is the Schwartzschild radius, $k_{q}$ is a characteristic length of the Reissner-Nordstrom spacetime and $q_{0}$ and $\hbar_{0}$ mean the numerical values of a charge $q$ and $\hbar$ with respect to the chosen units. Then

$$
\alpha^{0}=\left(\left|-\left(1-\frac{k_{s}}{r}+\frac{k_{q}^{2}}{r^{2}}\right)+\left(1-\frac{k_{s}}{r}+\frac{k_{q}^{2}}{r^{2}}\right)^{-1} r_{0}^{2}+r^{2} \theta_{0}^{2}+r^{2} \sin ^{2} \theta^{2} \varphi_{0}^{2}\right|\right)^{-1 / 2}
$$

$$
\begin{aligned}
\tau=-c^{-1} \alpha^{0}( & -\left(1-\frac{k_{s}}{r}+\frac{k_{q}^{2}}{r^{2}}\right) d t \\
& \left.+\left(1-\frac{k_{s}}{r}+\frac{k_{q}^{2}}{r^{2}}\right)^{-1} r_{0} d r+r^{2} \theta_{0} d \theta+r^{2} \sin ^{2} \theta \varphi_{0} d \varphi\right)
\end{aligned}
$$

and $G_{\perp i j}=\frac{m}{\hbar}\left(g_{i j}+\left(\alpha^{0}\right)^{2} g_{i i} g_{j j} x_{0}^{i} x_{0}^{j}\right)$ (no sum in $\left.i, j\right)$, where we set $g_{i j}=0$ if $i \neq j$. In this case the metric Christoffel symbols $K_{\rho}{ }^{\nu}{ }_{\sigma}$ are not all zero, their expression can be easily computed. It follows that

$$
\begin{aligned}
& \Omega^{\mathfrak{g}}=c_{0} \alpha^{0} \frac{m}{\hbar} \sum_{i, j=1}^{3}\left(g_{i j}+\left(\alpha^{0}\right)^{2} g_{i i} g_{j j} x_{0}^{i} x_{0}^{j}\right) \\
& \quad\left(d_{0}^{i}-\left(K_{\lambda{ }^{i}{ }^{i}}-x_{0}^{i} K_{\lambda}{ }^{0}{ }_{0}+x_{0}^{p} K_{\lambda}{ }^{i}{ }_{p}-x_{0}^{p} x_{0}^{i} K_{\lambda}{ }^{0}{ }_{p}\right) d^{\lambda}\right) \wedge \omega^{j} .
\end{aligned}
$$

Note that $\widehat{F}=2 d \widehat{A}=-\left(q_{0} /\left(\hbar_{0} r^{2}\right)\right) d^{0} \wedge d^{r}$. The Lagrangian is $L_{0}=$ $-(m c) /\left(\hbar \alpha^{0}\right)-\left(q_{0} / \hbar_{0} r\right)$. As before, $\boldsymbol{E}$ is parallelizable and there is a priviliged observer, with respect to which the Hamiltonian takes the form $\mathcal{H}[o]=$ $-\left((m c / \hbar) \alpha^{0} g_{00}-\left(q_{0} /\left(\hbar_{0} r\right)\right)\right) \bar{d}^{0}$. 


\section{Infinitesimal symmetries of spacetime and phase objects}

Symmetries of spacetime are diffeomorphisms which preserve the geometric structure of $\boldsymbol{E}$, i.e., the structure of pseudo-Riemannian manifold and its consequences. On the other hand symmetries of the phase space are fibered diffeomorphisms of the phase space which preserve geometrical objets on the phase space, the time form and contact mappings. These symmetries will be the subject of the first subsection. In the second subsection, we will consider symmetries of connections and in the third subsection symmetries of dynamical objects, i.e., the phase 2-form and the phase 2-vector.

\subsection{Spacetime and phase infinitesimal symmetries}

We can define 3 types of symmetries of the spacetime $\boldsymbol{E}$.

3.1 Definition. Let $f: \boldsymbol{E} \rightarrow \boldsymbol{E}$ be a diffeomorphism. Then $f$ is

1. a motion preserving symmetry of $\boldsymbol{E}$ if $f$ transforms any motion in a motion, i.e. $g_{f(x)}(T f(X), T f(X))<0$ for any timelike vector $X \in T_{x} \boldsymbol{E}$.

2. a time preserving symmetry of $\boldsymbol{E}$ if $f$ preserves the time form, i.e. $\tau\left(j_{1} s\right)(X)=\tau\left(j_{1}(f \circ s)\right)(T f(X))$.

3. a metric preserving symmetry of $\boldsymbol{E}$, or an isometry of $\boldsymbol{E}$, if $f$ is an isometry of the pseudo-Riemannian manifold $(\boldsymbol{E}, g)$.

Any symmetry in the sense 1 preserves the phase space, in the sense that it can be prolonged to a diffeomorphism $\partial_{1} f$ of $\mathcal{\partial}_{1} \boldsymbol{E}$. Any symmetry in the case 2 is a symmetry in the case 1 and any symmetry in the sense 3 is a symmetry in the case 2 and 1.

A vector field on $\boldsymbol{E}$ is said to be a motion (respective time, respective metric) preserving infinitesimal symmetry of $\boldsymbol{E}$ if its flow is a motion (respective time, respective metric) preserving symmetry of $\boldsymbol{E}$. Since $\mathcal{J}_{1} \boldsymbol{E}$ is defined by an 'open' condition (note that $\operatorname{dim} J_{1}(\boldsymbol{E}, 1)=\operatorname{dim} \partial_{1} \boldsymbol{E}$ ), every vector field on $J_{1}(\boldsymbol{E}, 1)$ restricts to a vector field on $\mathcal{J}_{1} \boldsymbol{E}$ whose flow evidently consists of motion preserving symmetries. This fact is stressed in the following Lemma.

3.2 Lemma. Any vector field $X$ on $\boldsymbol{E}$ is a motion preserving infinitesimal symmetry of $\boldsymbol{E}$, i.e. its flow is motion preserving.

Proof. $J_{1} \boldsymbol{E}$ is an open subspace in $J_{1}(\boldsymbol{E}, 1)$, so the holonomic lift $X_{(1)}$ of any vector field $X$ on $\boldsymbol{E}$ can be restricted to the vector field on $\mathcal{J}_{1} \boldsymbol{E}$, denoted by the same symbol $X_{(1)}$. Then its flow $F L_{t}^{X(1)}=\mathcal{J}_{1} F L_{t}^{X}$ is a diffeomorphism of $\mathcal{J}_{1} \boldsymbol{E}$ which is the lift of a motion preserving diffeomorphism of $\boldsymbol{E}$. Hence the flow of $X$ is motion preserving. QED 
3.3 Remark. The above Lemma shows a subtle difference between our approach and the approach in [11]: for the tangent lift of $X$ to $T \boldsymbol{E}$ to restrict to the pseudosphere bundle he needs $X$ to be an isometry. Our lift is different from the tangent lift (note also that $\operatorname{dim} T \boldsymbol{E}=8$ !), but we recover isometries by requiring projectability and the symmetry of the geometric structures of the phase space, see below.

3.4 Definition. Let $Y$ be a vector field on $\mathcal{J}_{1} \boldsymbol{E}$ projectable on a vector field $X$ on $\boldsymbol{E}$. Then:

1. $X$ is a time preserving infinitesimal symmetry of $\boldsymbol{E}$ if its flow preserves the time form, i.e. $L_{X_{(1)}} \tau=0$.

2. $X$ is a metric preserving infinitesimal symmetry (or a Killing vector field) of $\boldsymbol{E}$ if $X$ is an infinitesimal isometry of $g$, i.e. $L_{X} g=0$.

3. $Y$ is a phase infinitesimal symmetry

Of course, case 2 implies case 1.

In what follows we shall characterize phase infinitesimal symmetries of the basic phase objects, i.e. phase infinitesimal symmetries of д, $\tau, \theta, v_{g}$ and $g_{\|}$. It is remarkable that even if we allow for projectable infinitesimal symmetries we obtain that they must be the holonomic lifts of distinguished infinitesimal symmetries of $\boldsymbol{E}$.

3.5 Remark. In principle we could study more general infinitesimal symmetries; we consider this question in detail in Section 5 .

3.6 Lemma. 1. Let $\alpha$ be a 1 -form on $\boldsymbol{E}$. Then $д\lrcorner \alpha=0$ if and only if $\alpha=0$.

2. Let $\Phi$ be a spacetime 2-form. Then д $\lrcorner \Phi=0$ if and only if $\Phi=0$.

3. Let $\Psi$ be a $(0,2)$ symmetric tensor field on $\boldsymbol{E}$. Then $\Psi($ д, д) $=0$ if and only if $\Psi=0$.

Proof. Let $\alpha=\alpha_{\lambda} d^{\lambda}$. Then д $\lrcorner \alpha=c \alpha^{0}\left(\alpha_{0}+\alpha_{p} x_{0}^{p}\right)$ and д $\lrcorner \alpha=0$ if and only if $\alpha_{0}+\alpha_{p} x_{0}^{p}=0$ for all $x_{0}^{p}$, but, from $\alpha_{\lambda}: \boldsymbol{E} \rightarrow \mathbb{R}$, it is possible if and only if $\alpha_{\lambda}=0$. Hence 1 follows, and also 2 by a similar computation. If $\Psi=\Psi_{\lambda \mu} d^{\lambda} \otimes d^{\mu}$, then $\Psi($ д, Д) $=$ $\left(c \alpha^{0}\right)^{2}\left(\Psi_{00}+2 \Psi_{p 0} x_{0}^{p}+\Psi_{p q} x_{0}^{p} x_{0}^{q}\right)$, hence the result follows. QED

Now, we can characterize phase infinitesimal symmetries of basic phase objects.

3.7 Theorem. Let $Y$ be a projectable vector field on $\mathcal{J}_{1} \boldsymbol{E}$ over a vector field $X$ on $\boldsymbol{E}$. Then, the following conditions are equivalent:

(1) $X$ is a Killing vector field and $Y=X_{(1)}$.

(2) $Y$ is a phase infinitesimal symmetry of д, i.e. $L_{Y}$ д $=0$.

(3) $Y$ is a phase infinitesimal symmetry of $\tau$, i.e. $L_{Y} \tau=0$. 
(4) $Y$ is a phase infinitesimal symmetry of $\theta$, i.e. $L_{Y} \theta=0$.

(5) $Y$ is a phase infinitesimal symmetry of $v_{g}^{-1}$, i.e. $L_{Y} v_{g}^{-1}=0$.

(6) $Y$ is a phase infinitesimal symmetry of $g_{\|}$, i.e. $L_{Y} g_{\|}=0$.

Proof. (1) $\Leftrightarrow(2)$. We define the Lie derivative of д with respect to a projectable vector field $Y$ on $\mathcal{J}_{1} \boldsymbol{E}$, over $X$ on $\boldsymbol{E}$, as the generalized Lie derivative by

$$
L_{Y} \text { д }=T \text { до } Y-\mathcal{T}(X) \circ \text { д },
$$

where $\mathcal{T}(X)$ is considered as the vector field on $\mathbb{T}^{*} \otimes T \boldsymbol{E}$ obtained as the tensor product of the identity vector field on $\mathbb{T}^{*}$ and the tangent lift of $X$ to $T \boldsymbol{E}$. Then $L_{Y}$ д has the coordinate expression

$$
L_{Y} \text { д }=c \alpha^{0}\left[\frac{1}{2}\left(\alpha^{0}\right)^{2} \breve{\delta}_{0}^{\lambda} X^{\rho} \partial_{\rho} \hat{g}_{00}+Y_{0}^{p} \delta_{p}^{\lambda}+\left(\alpha^{0}\right)^{2} Y_{0}^{p} \breve{g}_{0 p} \breve{\delta}_{0}^{\lambda}-\breve{\delta}_{0}^{\rho} \partial_{\rho} X^{\lambda}\right] \partial_{\lambda}
$$

and, by taking into account the splitting (44), we can rewrite $L_{Y}$ д as

$$
\begin{aligned}
L_{Y} \text { Д }= & c\left(\alpha^{0}\right)^{3}\left(\frac{1}{2} X^{\rho} \partial_{\rho} \hat{g}_{00}+\breve{g}_{0 \rho} \breve{\delta}_{0}^{\sigma} \partial_{\sigma} X^{\rho}\right) D_{0} \\
& +c \alpha^{0}\left(Y_{0}^{i}-\breve{\delta}_{\rho}^{i} \breve{\delta}_{0}^{\sigma} \partial_{\sigma} X^{\rho}\right) N_{i} .
\end{aligned}
$$

So $L_{Y \text { д }}=0$ if and only if

$$
\begin{aligned}
c\left(\alpha^{0}\right)^{3} \frac{1}{2} \breve{\delta}_{0}^{\sigma} \breve{\delta}_{0}^{\omega}\left(X^{\rho} \partial_{\rho} g_{\sigma \omega}+g_{\rho \omega} \partial_{\sigma} X^{\rho}+g_{\sigma \rho} \partial_{\omega} X^{\rho}\right) & =0, \\
Y_{0}^{i}-\breve{\delta}_{\rho}^{i} \breve{\delta}_{0}^{\sigma} \partial_{\sigma} X^{\rho} & =0 .
\end{aligned}
$$

(2) is equivalent with $\left(L_{X} g\right)\left(\right.$ Д, Д) $=0$ and, by Lemma 3.6 with $L_{X} g=0$. Further, (3) is equivalent with $Y=X_{(1)}$.

$(1) \Leftrightarrow(3)$. From (3) we have

$$
\left.\left.L_{Y} \tau=-\frac{1}{c^{2}}\left(L_{Y} \text { Д }\right\lrcorner g+\text { Д }\right\lrcorner L_{X} g\right) .
$$

If $X$ is a Killing vector field and $Y=X_{(1)}$, then $L_{X} g=0$ and $L_{Y}$ д $=0$, i.e. $L_{Y} \tau=0$.

On the other hand let $L_{Y} \tau=0$. Then

$$
\left.\left.L_{Y} \text { д }\right\lrcorner g=- \text { д }\right\lrcorner L_{X} g
$$

and, by using the isomorphism $g^{\sharp}$, we get

$$
\left.L_{Y} \text { д }=-(\text { д }\lrcorner L_{X} g\right)^{\sharp}=-c \alpha^{0} \breve{\delta}_{0}^{\rho}\left(L_{X} g\right)_{\rho \sigma} g^{\sigma \lambda} \partial_{\lambda} .
$$

In the adapted coordinates

$$
\text { - } \left.(\text { д }\lrcorner L_{X} g\right)^{\sharp}=c\left(\alpha^{0}\right)^{3} \breve{\delta}_{0}^{\rho} \breve{\delta}_{0}^{\sigma}\left(L_{X} g\right)_{\rho \sigma} D_{0}-\left(c \alpha^{0}\right)^{2} \breve{\delta}_{0}^{\rho}\left(L_{X} g\right)_{\rho \sigma} \breve{g}^{\sigma i} N_{i} .
$$

Now, by comparing the д-horizontal parts of (1) and (5), we get $L_{X} g=0$, i.e. $X$ is a Killing vector field. By comparing the $\tau$-vertical parts of (11) and (5) we get $Y=X_{(1)}$.

$(1) \Leftrightarrow(4)$. We have $\theta=\operatorname{id}_{\mathcal{T}_{1,0} \boldsymbol{E}}-\tau \otimes$ д , i.e.

$$
L_{Y} \theta=-L_{Y} \tau \otimes \text { д }-\tau \otimes L_{Y} \text { д. }
$$

Let $X$ be a Killing vector field and $Y=X_{(1)}$, then $L_{Y} \tau=0$ and $L_{Y}$ д $=0$ which implies $L_{Y} \theta=0$. 

it on д,

On the other hand let $L_{Y} \theta=0$, then $\tau \otimes L_{Y}$ д $=-L_{Y} \tau \otimes$ д which implies, by evaluating

$$
L_{Y} \text { д }=-\left(L_{Y} \tau\right)(\text { д) д } .
$$

So, the $\tau$-vertical part of $L_{Y}$ д vanishes and by (1) $Y=X_{(1)}$. Then the above equality reduces to

$$
L_{X_{(1)}} \text { д }=-\left(L_{X_{(1)}} \tau\right)(\text { д) д }
$$

but, from (11) and (4), we obtain

$$
\left.L_{X_{(1)}} \text { д }=\frac{1}{2 c^{2}}\left(L_{X} g\right)\left(\text { д, д) д }, \quad L_{X_{(1)}} \tau=-\frac{1}{c^{2}}\left(L_{X_{(1)}} \text { д }\right\lrcorner g+\text { д }\right\lrcorner L_{X} g\right) .
$$

(6) then leads to $\left(L_{X} g\right)($ д, д) $=0$, i.e., by Lemma 3.6 $X$ is a Killing vector field.

$(1) \Leftrightarrow(5)$. We have the coordinate expression

$$
\begin{aligned}
L_{Y} v_{g}^{-1}= & -\frac{1}{c \alpha^{0}}\left[\frac{1}{2}\left(\alpha^{0}\right)^{2} \breve{\delta}_{\mu}^{i} X^{\rho} \partial_{\rho} \hat{g}_{00}+\left(\alpha^{0}\right)^{2} \breve{\delta}_{\mu}^{i} Y_{0}^{p} \breve{g}_{0 p}+Y_{0}^{i} \delta_{\mu}^{0}\right. \\
& \left.+\breve{\delta}_{\mu}^{p} \partial_{p}^{0} Y_{0}^{i}-\breve{\delta}_{\rho}^{i} \partial_{\mu} X^{\rho}\right] d^{\mu} \otimes \partial_{i}^{0}
\end{aligned}
$$

and, in the adapted coordinates, we get

$$
\begin{aligned}
L_{Y} v_{g}^{-1}= & -\frac{1}{c \alpha^{0}}\left[\left(Y_{0}^{i}-\breve{\delta}_{\rho}^{i} \breve{\delta}_{0}^{\mu} \partial_{\mu} X^{\rho}\right) N^{0}-c \alpha^{0}\left(Y_{0}^{i}-\breve{\delta}_{\rho}^{i} \breve{\delta}_{0}^{\mu} \partial_{\mu} X^{\rho}\right) \tau_{j} \omega^{j}\right. \\
& \left.+\left(\frac{1}{2}\left(\alpha^{0}\right)^{2} \delta_{j}^{i} X^{\rho} \partial_{\rho} \hat{g}_{00}+\left(\alpha^{0}\right)^{2} \delta_{j}^{i} Y_{0}^{p} \breve{g}_{0 p}+\partial_{j}^{0} Y_{0}^{i}-\breve{\delta}_{\rho}^{i} \partial_{j} X^{\rho}\right) \omega^{j}\right] \otimes \partial_{i}^{0} .
\end{aligned}
$$

Then $L_{Y} v_{g}^{-1}=0$ if and only if

$$
\begin{aligned}
Y_{0}^{i}-\breve{\delta}_{\rho}^{i} \breve{\delta}_{0}^{\mu} \partial_{\mu} X^{\rho} & =0, \\
-c \alpha^{0}\left(Y_{0}^{i}-\breve{\delta}_{\rho}^{i} \breve{\delta}_{0}^{\mu} \partial_{\mu} X^{\rho}\right) \tau_{j}+ & \\
+\left(\frac{1}{2}\left(\alpha^{0}\right)^{2} \delta_{j}^{i} X^{\rho} \partial_{\rho} \hat{g}_{00}+\left(\alpha^{0}\right)^{2} \delta_{j}^{i} Y_{0}^{p} \breve{g}_{0 p}+\partial_{j}^{0} Y_{0}^{i}-\breve{\delta}_{\rho}^{i} \partial_{j} X^{\rho}\right) & =0 .
\end{aligned}
$$

Then (9) is equivalent with $Y=X_{(1)}$ and (10) reduces to

$$
\frac{1}{2}\left(\alpha^{0}\right)^{2} \delta_{j}^{i} \breve{\delta}_{0}^{\rho} \breve{\delta}_{0}^{\tau}\left(X^{\sigma} \partial_{\sigma} g_{\rho \tau}+g_{\tau \sigma} \partial_{\rho} X^{\sigma}+g_{\rho \sigma} \partial_{\tau} X^{\sigma}\right)=0
$$

which is equivalent with $X$ to be a Killing vector field.

$(3) \Leftrightarrow(6)$. We have $g_{\|}=-c^{2} \tau \otimes \tau$, then

$$
L_{Y} g_{\|}=-c^{2}\left(L_{Y} \tau \otimes \tau+\tau \otimes L_{Y} \tau\right): \mathcal{\partial}_{1} \boldsymbol{E} \rightarrow \mathbb{L}^{2} \otimes T^{*} \boldsymbol{E} \otimes T^{*} \boldsymbol{E}
$$

and $L_{Y} \tau=0$ implies $L_{Y} g_{\|}=0$.

On the other hand $L_{Y} g_{\|}=0$ implies $L_{Y} \tau \otimes \tau+\tau \otimes L_{Y} \tau=0$. Now let us consider the splitting (4) of $T \boldsymbol{E}$ and the corresponding adapted base $\left(D_{0}, N_{i}\right)$. We have $\tau\left(D_{0}\right)=\frac{1}{c \alpha^{0}}$ and $\tau\left(N_{i}\right)=0$. Then

$$
\begin{aligned}
& 0=\left(L_{Y} \tau \otimes \tau+\tau \otimes L_{Y} \tau\right)\left(D_{0}, D_{0}\right)=\frac{2}{c \alpha^{0}}\left(L_{Y} \tau\right)\left(D_{0}\right), \\
& 0=\left(L_{Y} \tau \otimes \tau+\tau \otimes L_{Y} \tau\right)\left(D_{0}, N_{i}\right)=\frac{1}{c \alpha^{0}}\left(L_{Y} \tau\right)\left(N_{i}\right),
\end{aligned}
$$

i.e. $L_{Y} \tau=0$ and $Y$ is an infinitesimal symmetry of $\tau$. QED 


\subsection{Infinitesimal symmetries of connections}

Let us recall that linear spacetime connections $K$ on $\boldsymbol{E}$ can be regarded as sections of the natural bundle $\mathcal{K}: Q \boldsymbol{E} \rightarrow \boldsymbol{E}$ of linear connections. The bundle $Q \boldsymbol{E}$ is defined as the affine subbundle of the bundle of linear morphisms $K: T \boldsymbol{E} \times_{\boldsymbol{E}} T \boldsymbol{E} \rightarrow T T \boldsymbol{E}$ over id $\mathrm{id}_{T \boldsymbol{E}}$ such that $\left(\tau_{T \boldsymbol{E}}, T \tau_{\boldsymbol{E}}\right) \circ K=\mathrm{id}_{T \boldsymbol{E} \times \times_{\boldsymbol{E}} T \boldsymbol{E}}$. The Lie derivative of $K$, with respect to a vector field $X$ on $\boldsymbol{E}$, is then the vertical vector field on $Q \boldsymbol{E}$ defined as

$$
L_{X} K=T K \circ X-\mathcal{Q}(X),
$$

where $\mathcal{Q}(X)$ is the flow lift, [21, p. 59], of $X$ on $Q \boldsymbol{E}$. Note that the vertical subspace of $T Q \boldsymbol{E}$ is defined as $V Q \boldsymbol{E}=\operatorname{ker} T \mathcal{K}$. In this way we obtain the standard Lie derivative with the coordinate expression

$$
\begin{array}{r}
L_{X} K=\left(X^{\rho} \partial_{\rho} K_{\mu}{ }^{\lambda}{ }_{\nu}-K_{\mu}{ }^{\rho}{ }_{\nu} \partial_{\rho} X^{\lambda}+K_{\rho}{ }_{\nu}{ }_{\nu} \partial_{\mu} X^{\rho}+K_{\mu}{ }^{\lambda}{ }_{\rho} \partial_{\nu} X^{\rho}-\partial_{\mu \nu}^{2} X^{\lambda}\right) \\
d^{\mu} \otimes \partial_{\lambda} \otimes d^{\nu}
\end{array}
$$

where we used the natural identification $V Q \boldsymbol{E} \simeq T^{*} \boldsymbol{E} \otimes T \boldsymbol{E} \otimes T^{*} \boldsymbol{E}$.

Let us note that if $X$ is an infinitesimal symmetry of $g$, then, by naturality property, $X$ is the infinitesimal symmetry of $K^{\mathfrak{g}}$.

3.8 Remark. Let us remark that we can define generally infinitesimal symmetries of $K$ as projectable vector fields $Y$ on $T \boldsymbol{E}$, over $X$ on $\boldsymbol{E}$, such that $L_{Y} K:=T K \circ Y-\left(\mathcal{T}^{*}(X) \otimes \mathcal{T}(Y)\right) \circ K=0$. Then we have two distinguished cases. First, if $Y=\mathcal{T}(X)$, then $L_{\mathcal{T}(X)} K$ is identified with $L_{X} K$ in the above sense. Second, if $Y=h^{K}(X)$, where $h^{K}(X)$ is the horizontal lift of $X$ with respect to the connection $K$, then $L_{h^{K}(X)} K=0$ if and only if $\left.X\right\lrcorner R=0$ where $R$ is the curvature tensor of $K$.

3.9 Remark. Let us note that any spacetime connection is of the form $K=K^{\mathfrak{g}}+\Phi$ where $\Phi$ is a symmetric $(1,2)$-tensor field on $\boldsymbol{E}$. Then a Killing vector field is an infinitesimal symmetry of $K$ if and only if it is an infinitesimal symmetry of $\Phi$.

We define the Lie derivative of $\Gamma$ with respect to a projectable vector field $Y$ on $\mathcal{J}_{1} \boldsymbol{E}$, over $X$ on $\boldsymbol{E}$, by

$$
L_{Y} \Gamma=T \Gamma \circ Y-\left(\mathcal{T}^{*}(X) \otimes \mathcal{T}(Y)\right) \circ \Gamma: \mathcal{J}_{1} \boldsymbol{E} \rightarrow T^{*} \boldsymbol{E} \otimes V_{\boldsymbol{E}} \mathcal{J}_{1} \boldsymbol{E},
$$

in coordinates, $L_{Y} \Gamma=\left(X^{\rho} \partial_{\rho} \Gamma_{\lambda_{0}}^{i}+Y_{0}^{p} \partial_{p}^{0} \Gamma_{\lambda_{0}}^{i}+\Gamma_{\rho_{0}}^{i} \partial_{\lambda} X^{\rho}-\partial_{\lambda} Y_{0}^{i}-\Gamma_{\lambda_{0}}^{p} \partial_{p}^{0} Y_{0}^{i}\right) d^{\lambda} \otimes$ $\partial_{i}^{0}$. From $\nu[\Gamma]=\mathrm{id}_{T d_{1} E}-\Gamma$ we have $L_{Y} \nu[\Gamma]=-L_{Y} \Gamma$, i.e. $L_{Y} \Gamma=0$ if and only if $L_{Y} \nu[\Gamma]=0$. Note that infinitesimal symmetries of $\Gamma$ and $v_{g}[\Gamma]$ are not 
equivalent: $L_{Y} v_{g}[\Gamma]=L_{Y} v_{g} \circ \nu[\Gamma]+v_{g} \circ L_{Y} \nu[\Gamma]$, so $L_{Y} v_{g}=0$ and $L_{Y} \Gamma=0$ imply $L_{Y} v_{g}[\Gamma]=0$. Since $L_{X_{(1)}} \chi(K)=\breve{\delta}_{\sigma}^{i} \breve{\delta}_{0}^{\rho}\left(L_{X} K\right)_{\lambda}{ }^{\sigma}{ }_{\rho} d^{\lambda} \otimes \partial_{i}^{0}$, we obtain the following result.

3.10 Proposition. Let $K$ be a spacetime connection. Let $X$ be a vector field on $\boldsymbol{E}$ and $X_{(1)}$ the corresponding holonomic lift. Then

$$
L_{X} K=0 \quad \Longrightarrow \quad L_{X_{(1)}} \chi(K)=0 \text {. }
$$

In particular, any Killing vector field $X$ fulfills $L_{X} K^{\mathfrak{g}}=0, L_{X_{(1)}} \Gamma^{\mathfrak{g}}=0$, $L_{X_{(1)}} \gamma^{\mathfrak{g}}=0$.

Let us remark that any phase connection is of the form $\Gamma=\Gamma^{\mathfrak{g}}+\Sigma$, where $\Sigma: \mathcal{J}_{1} \boldsymbol{E} \rightarrow T^{*} \boldsymbol{E} \otimes V_{\boldsymbol{E}} \mathcal{J}_{1} \boldsymbol{E}$ is a vertical valued 1-form. Then it is easy to see that for a Killing vector field $L_{X_{(1)}} \Gamma=0$ if and only if $L_{X_{(1)}} \Sigma=0$. Moreover, if $Y$ is an infinitesimal symmetry of д then $L_{Y} \Gamma=0$ implies $L_{Y} \gamma[\Gamma]=0$. In fact, $L_{Y} \gamma[\Gamma]=0$ if and only if $L_{Y}$ д $\lrcorner \Gamma=-$ д $\lrcorner L_{Y} \Gamma$.

3.11 Theorem. Let $X$ be a Killing vector field and $\Gamma=\Gamma^{\mathfrak{g}}+\Gamma^{\mathfrak{e}}$ the total phase connection. Then the following conditions are equivalent:

$$
L_{X_{(1)}} \Gamma=0 \quad \Leftrightarrow \quad L_{X_{(1)}} v_{g}[\Gamma]=0 \quad \Leftrightarrow \quad L_{X} \widehat{F}=0 \quad \Leftrightarrow \quad L_{X_{(1)}} \gamma[\Gamma]=0 .
$$

Proof. For the joined phase connection $\Gamma=\Gamma^{\mathfrak{g}}+\Gamma^{\mathfrak{e}}$ we have $L_{X_{(1)}} \Gamma=0$ if and only if $L_{X_{(1)}} \Gamma^{\mathfrak{e}}=0$. Similarly $L_{X_{(1)}} v_{g}[\Gamma]=0$ is equivalent with $L_{X_{(1)}} \nu\left[\Gamma^{\mathfrak{e}}\right]=0$, i.e. $L_{X_{(1)}} \Gamma^{\mathfrak{e}}=0$. So we have to prove that $L_{X_{(1)}} \nu\left[\Gamma^{\mathfrak{e}}\right]=0$ is equivalent with $L_{X} \widehat{F}=0$. field

We have $\Gamma^{\mathfrak{e}}=-\frac{1}{2} v_{g}^{-1} \circ G^{\sharp 2} \circ(\widehat{F}+2 \tau \wedge($ д $\widehat{F}))$ which implies that for a Killing vector

$$
\left.L_{X_{(1)}} \Gamma^{\mathfrak{e}}=-\frac{1}{2}\left(v_{g}^{-1} \circ G^{\sharp 2}\right)\left(L_{X} \widehat{F}+2 \tau \wedge(\text { д }\lrcorner L_{X} \widehat{F}\right)\right) .
$$

Then $L_{X} \widehat{F}=0$ implies $L_{X_{(1)}} \Gamma^{\mathfrak{e}}=0$ and $L_{X_{(1)}} v_{g}[\Gamma]=0$.

On the other hand $L_{X} \widehat{F}+2 \tau \wedge($ д $\left.\lrcorner L_{X} \widehat{F}\right): \mathcal{J}_{1} \boldsymbol{E} \rightarrow T^{*} \boldsymbol{E} \wedge V_{1,0}^{*} \boldsymbol{E}$ which implies that $\left(v_{g}^{-1} \circ G^{\sharp 2}\right)\left(L_{X} \widehat{F}+2 \tau \wedge(\right.$ д $\left.\lrcorner L_{X} \widehat{F}\right)=0$ if and only if $L_{X} \widehat{F}+2 \tau \wedge($ д $\left.\lrcorner L_{X} \widehat{F}\right)=0$. Evaluating values on the adapted base $\left(D_{0}, N_{i}\right)$ we get

$$
\begin{aligned}
& \left.0=\left(L_{X} \widehat{F}+2 \tau \wedge(\text { д }\lrcorner L_{X} \widehat{F}\right)\right)\left(D_{0}, D_{0}\right)=\left(L_{X} \widehat{F}\right)\left(D_{0}, D_{0}\right), \\
& \left.0=\left(L_{X} \widehat{F}+2 \tau \wedge(\text { д }\lrcorner L_{X} \widehat{F}\right)\right)\left(D_{0}, N_{i}\right)=2\left(L_{X} \widehat{F}\right)\left(D_{0}, N_{i}\right), \\
& \left.0=\left(L_{X} \widehat{F}+2 \tau \wedge(\text { д }\lrcorner L_{X} \widehat{F}\right)\right)\left(N_{i}, N_{j}\right)=\left(L_{X} \widehat{F}\right)\left(N_{i}, N_{j}\right),
\end{aligned}
$$

hence $L_{X_{(1)}} \Gamma^{\mathfrak{e}}=0$ implies $L_{X} \widehat{F}=0$.

The implication $L_{X_{(1)}} \Gamma=0 \Rightarrow L_{X_{(1)}} \gamma[\Gamma]=0$ follows from the remarks preceding this Theorem. On the other hand

$$
\begin{aligned}
0 & \left.\left.=L_{X_{(1)}} \gamma[\Gamma]=L_{X_{(1)}} \gamma\left[\Gamma^{\mathfrak{e}}\right]=\text { д }\right\lrcorner L_{X_{(1)}} \Gamma^{\mathfrak{e}}=-\frac{1}{2} \text { д }\right\lrcorner\left(v_{g}^{-1} \circ G^{\sharp 2}\right)\left(L_{X} \widehat{F}+2 \tau \wedge(\text { д }\lrcorner L_{X} \widehat{F}\right) \\
& \left.=-\left(v_{g}^{-1} \circ G^{\sharp}\right)(\text { д }\lrcorner L_{X} \widehat{F}\right) .
\end{aligned}
$$


I.e. $L_{X_{(1)}} \gamma[\Gamma]=0$ is equivalent with $\left.д\right\lrcorner L_{X} \widehat{F}=0$ and, by Lemma 3.6, with $L_{X} \widehat{F}=0$. So $L_{X_{(1)}} \gamma[\Gamma]=0$ is equivalent with $L_{X} \widehat{F}=0$ which implies, by Theorem 3.11, $L_{X_{(1)}} \Gamma=0$. QED

3.12 Remark. Let us note that $\gamma^{\mathfrak{g}}$ is a scaled (non projectable) infinitesimal symmetry of $\tau$. Really, we have $i_{\gamma^{\mathfrak{g}}} \tau=1$ and $d \tau=-\frac{\hbar}{m c^{2}} \Omega^{\mathfrak{g}}$, Then $L_{\gamma^{\mathfrak{g}}} \tau=i_{\gamma^{\mathfrak{g}}} d \tau+d i_{\gamma^{\mathfrak{g}}} \tau=-\frac{\hbar}{m c^{2}} i_{\gamma^{\mathfrak{g}}} \Omega^{\mathfrak{g}}+d(1)=0$.

\subsection{Infinitesimal symmetries of dynamical objects}

Now, let $\Omega=\Omega[G, \Gamma]$ be the joined phase 2 -form and $\Lambda=\Lambda[G, \Gamma]$ be the joined phase 2 -vector given by the rescaled metric $G$ and the joined phase connection $\Gamma$. We define an infinitesimal symmetry of $\Omega$ to be a vector field $Y$ on $\mathcal{\partial}_{1} \boldsymbol{E}$ such that $L_{Y} \Omega=0$. Let us note that the joined $\gamma$ is a (scaled non projectable) infinitesimal symmetry of $\Omega$. A phase function $f: \partial_{1} \boldsymbol{E} \rightarrow \mathbb{R}$ such that $\gamma . f=0$ is said to be conserved.

3.13 Theorem. Infinitesimal symmetries $Y: \mathcal{J}_{1} \boldsymbol{E} \rightarrow T_{\mathcal{J}_{1}} \boldsymbol{E}$ of $\Omega$ are of local type $Y=\gamma(\kappa)+d f^{\sharp}$, where $\kappa=\tau(Y): \mathcal{J}_{1} \boldsymbol{E} \rightarrow \overline{\mathbb{T}}$ and $f: \mathcal{J}_{1} \boldsymbol{E} \rightarrow \mathbb{R}$ is a conserved phase function determined up to a constant.

Proof. Let us consider a vector field $Y: \mathfrak{J}_{1} \boldsymbol{E} \rightarrow T \mathcal{J}_{1} \boldsymbol{E}$. Then, according to the tangent splitting $T \mathcal{J}_{1} \boldsymbol{E}=H_{\gamma} \mathcal{J}_{1} \boldsymbol{E} \oplus V_{\tau} \mathcal{J}_{1} \boldsymbol{E}$, we have $Y=\gamma(\kappa)+\bar{Y}$, where $\tau(\bar{Y})=0$.

Then, by recalling the closure of $\Omega$ and $i_{\gamma} \Omega=0$, we have $L_{Y} \Omega=d i_{Y} \Omega=d i_{\bar{Y}} \Omega$. I.e. $Y$ is an infinitesimal symmetry of $\Omega$ if and only if $d i_{\bar{Y}} \Omega=0$ and hence, locally, $i_{\bar{Y}} \Omega=d f$ for a phase function $f$ and, moreover, $0=i_{\bar{Y}} i_{\gamma} \Omega=i_{\gamma} i_{\bar{Y}} \Omega=i_{\gamma} d f=\gamma \cdot f$. Then $\bar{Y}=d f^{\sharp}$ and $Y=\gamma(\kappa)+d f^{\sharp}$. QED

Infinitesimal symmetries of $\Lambda$ are vector fields $Y$ on $\mathcal{J}_{1} \boldsymbol{E}$ such that $L_{Y} \Lambda=$ $[Y, \Lambda]=0$. It is easy to realize that, in general, infinitesimal symmetries of $\Omega$ and $\Lambda$ are not equivalent, even in case of $Y$ projectable; they are equivalent only if we assume that $Y=X_{(1)}$ for a Killing vector field $X$.

3.14 Theorem. Let $\Gamma$ be a phase connection, $X$ be a Killing vector field and $X_{(1)}$ the corresponding holonomic lift. Then

$$
\begin{gathered}
L_{X_{(1)}} \Gamma=0 \quad \Longrightarrow \quad L_{X_{(1)}} \Omega=0, \quad L_{X_{(1)}} \Lambda=0, \\
L_{X_{(1)}} \Omega=0 \quad \Longleftrightarrow \quad L_{X_{(1)}} \Lambda=0 .
\end{gathered}
$$

Proof. For a Killing vector field $X$, we get $\left.L_{X_{(1)}} \Omega=G\right\lrcorner\left(\left(v_{g} \circ L_{X_{(1)}} \nu[\Gamma]\right) \wedge \theta\right)$, hence $L_{X_{(1)}} \Gamma=-L_{X_{(1)}} \nu[\Gamma]=0$ implies $L_{X_{(1)}} \Omega=0$, and the same for $\Lambda$.

It is easy to see that $L_{X_{(1)}} \Lambda=-\left(\Lambda^{\sharp} \otimes \Lambda^{\sharp}\right)\left(L_{X_{(1)}} \Omega\right), L_{X_{(1)}} \Omega=-\left(\Omega^{b} \otimes \Omega^{b}\right)\left(L_{X_{(1)}} \Lambda\right)$, which yields the result. QED 
In view of Proposition 3.10, if $K$ is a spacetime connection and $X$ be a Killing vector field, then $L_{X} K=0$ implies $L_{X_{(1)}} \chi(K)=0$, hence $L_{X_{(1)}} \Omega=0$ and $L_{X_{(1)}} \Lambda=0$. Moreover, $L_{X_{(1)}} \Omega^{\mathfrak{g}}=0, L_{X_{(1)}} \Lambda^{\mathfrak{g}}=0$.

As a consequence of the above theorem, we can prove that symmetries of dynamical structures are also symmetries of the Lagrangian and the EulerLagrange morphism.

3.15 Theorem. Let $\Gamma$ be a phase connection, $X$ be a Killing vector field and $X_{(1)}$ the corresponding holonomic lift. Then

1. $L_{X_{(1)}} \Gamma=0 \quad \Longrightarrow \quad L_{X_{(1)}} \eta=0 \quad \Longleftrightarrow \quad L_{X_{(1)}} \mathcal{L}=h(d f)$;

2. $L_{X_{(1)}} \mathcal{L}=0 \quad \Longleftrightarrow \quad L_{X_{(1)}} \Theta=0$;

3. given an observer $o$ such that $L_{X_{(1)}} o=0$ then $L_{X_{(1)}} \mathcal{H}[o]=0 \Longleftrightarrow$ $L_{X_{(1)}} \mathcal{L}=0$.

Proof. 1. Recall that $\eta=\bar{g}_{\perp}(\nabla[\gamma])$. By $L_{X_{(1)}} \gamma[\Gamma]=0$ (Theorem 3.11) and by $\bar{g}_{\perp}=\bar{g}(\theta, \theta)$, being $L_{X_{(1)}} \theta=0$ we have $L_{X_{(1)}} \eta=0$. The second part follows from the well-known fact 3,29 , that the Euler-Lagrange operator $\mathcal{E}$ commutes with the Lie derivative: $\mathcal{E}\left(L_{X_{(1)}} \mathcal{L}\right)=L_{X_{(1)}} \eta$. This implies that a symmetry of $\eta$ is a divergence symmetry of $\mathcal{L}$, i.e., symmetry up to a total divergence, which is the horizontalization of a closed form.

2. Lie derivative with respect to a Killing vector field preserves the splitting (44). Hence $L_{X_{(1)}} h(\Theta)=h\left(L_{X_{(1)}} \Theta\right)=\left(L_{X_{(1)}} \Theta\right)($ д). Then, Lemma 3.6 yields the result.

3. It is a straightforward consequence of the above facts.

\section{Phase infinitesimal symmetries and special phase functions}

From now we assume the almost-cosymplectic-contact structure and the dual almost-coPoisson-Jacobi structure on the phase space given by the pairs $(-\widehat{\tau}, \Omega)$ and $(-\widehat{\gamma}, \Lambda)$. A phase infinitesimal symmetry of the classical phase structure is defined to be a projectable vector field $Y$ on $\mathcal{J}_{1} \boldsymbol{E}$ which is an infinitesimal symmetry of $\widehat{\tau}$ and $\Omega$. We recall that, due to Lemma 1.1, phase infinitesimal symmetries of $(-\widehat{\tau}, \Omega)$ and $(-\widehat{\gamma}, \Lambda)$ are the same.

\subsection{Special phase functions}

In Theorem 3.13 if we assume that $Y$ is a projectable vector field over a vector field $X$ on $\boldsymbol{E}$ then in the expression $Y=\gamma(\kappa)+d f^{\sharp}$ the phase function $f$ has 
to be a special phase function, and $Y$ is the so called special Hamiltonian lift $X^{\uparrow}[f]$ of $f,[15] . f$ is said to be a generator of the infinitesimal symmetry $Y$ of $\Omega$.

We recall [15] that special phase functions are of the type

$$
f=-G(\text { д, } X)+\breve{f}=\widehat{\tau}(X)+\breve{f},
$$

where $X$ is a vector field on $\boldsymbol{E}$ and $\breve{f}$ is a spacetime function. So, the special phase function $f$ can be identified with the pair $(X, \breve{f})$. We have the coordinate expression $f=-c_{0} \alpha^{0} \breve{G}_{0 \rho}^{0} X^{\rho}+\breve{f}$. We denote by $\operatorname{spec}\left(\mathcal{J}_{1} \boldsymbol{E}, \mathbb{R}\right)$ the sheaf of special phase functions.

The special Hamiltonian lift of a special phase function with respect to the joined almost-coPoisson-Jacobi pair $(-\widehat{\gamma}, \Lambda)$ is

$$
X^{\uparrow}[f]=H[f]-\breve{f} \widehat{\gamma}=d f^{\sharp}+(f-\breve{f}) \widehat{\gamma}=d f^{\sharp}+\widehat{\tau}(X) \widehat{\gamma},
$$

where $H[f]=d f^{\sharp}+f \widehat{\gamma}$ is the standard Hamiltonian lift. We have the coordinate expression

$$
X^{\uparrow}[f]=X^{\lambda} \partial_{\lambda}-\breve{G}_{0}^{i \sigma}\left(-\frac{1}{c_{0} \alpha^{0}} \partial_{\sigma} \breve{f} X^{\rho} \partial_{\rho} \breve{G}_{0 \sigma}^{0}+\breve{G}_{0 \rho}^{0} \partial_{\sigma} X^{\rho}+\frac{1}{c_{0} \alpha^{0}} X^{\rho} \widehat{F}_{\rho \sigma}\right) \partial_{i}^{0} .
$$

For a phase function $f=-G($ д, $X)$ corresponding to a vector field $X$ on $\boldsymbol{E}$ the special Hamiltonian lift coincides with the standard Hamiltonian lift, i.e. $X^{\uparrow}[f]=H[f]$. For a spacetime function $f$ the special Hamiltonian lift is a vertical vector field.

Let us recall that we define the special bracket [15] of two special phase functions $f=-G($ д, $X)+\breve{f}$ and $h=-G\left(\right.$ д, $\left.X^{\prime}\right)+\breve{h}$ to be

$$
\llbracket f, h \rrbracket=[f, h]-(\breve{f} \widehat{\gamma} \cdot h-\breve{h} \widehat{\gamma} \cdot f),
$$

where [,] is the Jacobi bracket. We can rewrite the special brackets as

$$
\begin{aligned}
\llbracket f, h \rrbracket & =\{f, h\}+((f-\breve{f}) \widehat{\gamma} \cdot h-(h-\breve{h}) \widehat{\gamma} \cdot f) \\
& =\{f, h\}+\widehat{\tau}(X) \widehat{\gamma} \cdot h-\widehat{\tau}\left(X^{\prime}\right) \widehat{\gamma} \cdot f,
\end{aligned}
$$

where $\{f, h\}=\Lambda(d f, d h)$ is the Poisson bracket. The sheaf $\operatorname{spec}\left(\mathcal{J}_{1} \boldsymbol{E}, \mathbb{R}\right)$ is closed with respect to the special bracket [15]. Indeed,

$$
\llbracket f, h \rrbracket=-G\left(\text { д, }\left[X, X^{\prime}\right]\right)+X . \breve{h}-X^{\prime} . \breve{f}+\widehat{F}\left(X, X^{\prime}\right)
$$

which is a special phase function given by the pair $\left(\left[X, X^{\prime}\right], X . \breve{h}-X^{\prime} . \breve{f}+\right.$ $\left.\widehat{F}\left(X, X^{\prime}\right)\right)$. 


\subsection{Special phase functions and infinitesimal symme- tries of dynamical objects}

The special Hamiltonian lift of a special phase function $f$ is an infinitesimal symmetry of $\Omega$ if and only if $f$ is conserved. Let us analyze more in detail this correspondence.

4.1 Theorem. The sheaf of conserved special phase functions is closed with respect to the special bracket, and the special Hamiltonian lift is a Lie algebra isomorphism from the Lie algebra of conserved special phase functions to the Lie algebra of projectable infinitesimal symmetries of $\Omega$.

Proof. If $\widehat{\gamma} \cdot f=0$ and $\widehat{\gamma} \cdot h=0$ then $\llbracket f, h \rrbracket=\{f, h\}$. Theorem then follows from

$$
\widehat{\gamma} \cdot\{f, h\}=\{\widehat{\gamma} \cdot f, h\}+\{f, \widehat{\gamma} \cdot h\}=0 .
$$

Indeed, $\widehat{\gamma} \cdot\{f, h\}=\left(L_{\widehat{\gamma}} \Lambda\right)(d f, d h)+\Lambda\left(L_{\widehat{\gamma}} d f, d h\right)+\Lambda\left(d f, L_{\widehat{\gamma}} d h\right)=-\left(\widehat{\gamma} \wedge\left(L_{\widehat{\gamma}} \widehat{\tau}\right)^{\sharp}\right)(d f, d h)+$

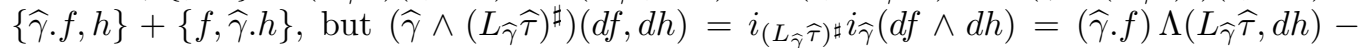
$(\widehat{\gamma} \cdot h) \Lambda\left(L_{\widehat{\gamma}} \widehat{\tau}, d f\right)=0$.

We have, [18,

$$
\left.\left.X^{\uparrow}[\llbracket f, h \rrbracket]=\left[X^{\uparrow}[f], X^{\uparrow}[h]\right]+\left((\widehat{\gamma} \cdot f)\left(d \breve{h}-X^{\prime}\right\lrcorner \widehat{F}\right)-(\widehat{\gamma} \cdot h)(d \breve{f}-X\lrcorner \widehat{F}\right)\right)^{\sharp} .
$$

So for conserved special phase functions $X^{\uparrow}[\llbracket f, h \rrbracket]=\left[X^{\uparrow}[f], X^{\uparrow}[h]\right]$. QED

4.2 Proposition. A special phase function $f=-G($ д, $X)+\breve{f}$ is conserved if and only if

$$
\text { д. } \breve{f}-(X\lrcorner \widehat{F})(\text { д })=\frac{1}{2}\left(L_{X} G\right)(\text { д, д) . }
$$

Proof. We can calculate it in coordinates, by using the identity $\breve{G}_{0}^{i \sigma} \breve{G}_{i \rho}^{0}=\delta_{\rho}^{\sigma}+$ $\left(\alpha^{0}\right)^{2} \breve{g}_{0 \rho} \breve{\delta}_{0}^{\sigma}$

$$
\begin{aligned}
\gamma \cdot f & =i_{\gamma} d f=c \alpha^{0} \breve{\delta}_{0}^{\lambda}\left(\partial_{\lambda} \breve{f}+\widehat{F}_{\lambda \sigma} X^{\sigma}-c_{0} \alpha^{0} \frac{1}{2} \breve{\delta}_{0}^{\mu}\left(X^{\rho} \partial_{\rho} G_{\lambda \mu}^{0}+G_{\rho \mu}^{0} \partial_{\lambda} X^{\rho}+G_{\lambda \rho}^{0} \partial_{\mu} X^{\rho}\right)\right) \\
& \left.\left.=\text { д }\lrcorner(d \breve{f}-X\lrcorner \widehat{F}-\frac{1}{2} \text { д }\right\lrcorner L_{X} G\right) . \text { QED }
\end{aligned}
$$

The above proposition implies that, if $f=-G($ д, $X)+\breve{f} \in \operatorname{spec}\left(\mathcal{J}_{1} \boldsymbol{E}, \mathbb{R}\right)$ where $X$ is a Killing vector field, then $f$ is conserved if and only if д. $\breve{f}-$ $(X\lrcorner \widehat{F})($ д $)=0$, i.e. if and only if $d \breve{f}=X\lrcorner \widehat{F}$. This also implies that a spacetime function $\breve{f}$ is conserved if and only if it is constant.

4.3 Lemma. Let $X$ be a Killing vector field of $\boldsymbol{E}$ and $f=-G($ д, $X)+\breve{f}$ a corresponding conserved special phase function. Then

$$
X^{\uparrow}[f]=X_{(1)} .
$$


Proof. We have

$$
\begin{aligned}
X^{\uparrow}[f] & =X^{\lambda} \partial_{\lambda}-\breve{G}_{0}^{i \sigma}\left(-\frac{1}{c_{0} \alpha^{0}} \partial_{\sigma} \breve{f}+X^{\rho} \partial_{\rho} \breve{G}_{0 \sigma}^{0}+\breve{G}_{0 \rho}^{0} \partial_{\sigma} X^{\rho}+\frac{1}{c_{0} \alpha^{0}} X^{\rho} \widehat{F}_{\rho \sigma}\right) \partial_{i}^{0} \\
& =X^{\lambda} \partial_{\lambda}+\breve{G}_{0}^{i \sigma} \breve{\delta}_{0}^{\tau} \breve{G}_{\sigma \rho}^{0} \partial_{\tau} X^{\rho} \partial_{i}^{0}=X^{\lambda} \partial_{\lambda}+\left(\delta_{\rho}^{i}-x_{0}^{i} \delta_{\rho}^{0}\right) \breve{\delta}_{0}^{\tau} \partial_{\tau} X^{\rho} \partial_{i}^{0} \\
& =X^{\lambda} \partial_{\lambda}+\breve{\delta}_{\rho}^{i} \breve{\delta}_{0}^{\tau} \partial_{\tau} X^{\rho} \partial_{i}^{0}=X_{(1)} \cdot \mathrm{QED}
\end{aligned}
$$

Now, let us consider a special phase function $f$ satisfying $X\lrcorner \widehat{F}=d \breve{f}$. Such a function generates an infinitesimal symmetry of $\widehat{F}: L_{X} \widehat{F}=0$.

4.4 Proposition. Let $f$ and $h$ be special phase functions given by $(X, \breve{f})$, $\left(X^{\prime}, \breve{h}\right)$ respectively, and let $\left.\left.X\right\lrcorner \widehat{F}=d \breve{f}, X^{\prime}\right\lrcorner \widehat{F}=d \breve{h}$. Then

$$
\left.d\left(X . \breve{h}-X^{\prime} . \breve{f}+\widehat{F}\left(X, X^{\prime}\right)\right)=\left[X, X^{\prime}\right]\right\lrcorner \widehat{F},
$$

so that the special bracket of $f$ and $h$ generates an infinitesimal symmetry of $\widehat{F}$.

Proof. We have

$$
\begin{aligned}
d\left(X . \breve{h}-X^{\prime} . \breve{f}+\widehat{F}\left(X, X^{\prime}\right)\right) & \left.\left.\left.\left.\left.\left.=d(X\lrcorner X^{\prime}\right\lrcorner \widehat{F}-X^{\prime}\right\lrcorner X\right\lrcorner \widehat{F}+X^{\prime}\right\lrcorner X\right\lrcorner \widehat{F}\right) \\
& =-d\left(\widehat{F}\left(X, X^{\prime}\right)\right) .
\end{aligned}
$$

On the other hand

$$
\begin{aligned}
{\left.\left[X, X^{\prime}\right]\right\lrcorner \widehat{F} } & \left.=\frac{1}{2} i_{\left[X, X^{\prime}\right]} \widehat{F}=\frac{1}{2} L_{X} i_{X^{\prime}} \widehat{F}=\left(d i_{X}+i_{X} d\right)\left(X^{\prime}\right\lrcorner \widehat{F}\right) \\
& \left.\left.=d(X\lrcorner X^{\prime}\right\lrcorner \widehat{F}\right)+i_{X} d d \breve{h}=-d\left(\widehat{F}\left(X, X^{\prime}\right)\right) \cdot \text { QED }
\end{aligned}
$$

We shall call special phase functions satisfying the above properties electromagnetic special phase functions.

4.5 Proposition. If $f$ is a conserved electromagnetic special phase function, then its corresponding vector field is a Killing vector.

Proof. If д. $\breve{f}-(X\lrcorner \widehat{F})\left(\right.$ д) $=\frac{1}{2}\left(L_{X} G\right)($ д, д) and $X\lrcorner \widehat{F}=d \breve{f}$ we get $\left(L_{X} G\right)($ д, д) $=0$, i.e. $L_{X} G=0$, and conversely, due to Proposition 4.2

QED

\subsection{Holonomic special phase functions and infinitesi- mal symmetries}

We define the sheaf of special holonomic phase functions to be the subsheaf of $f \in \operatorname{spec}\left(\mathcal{J}_{1} \boldsymbol{E}, \mathbb{R}\right)$ such that $X^{\uparrow}[f]=X_{(1)}$. 
4.6 Theorem. Let $X$ be a Killing vector field of $\boldsymbol{E}$ and $f=-G($ д, $X)+\breve{f}$ a corresponding special phase function. Then $f$ is conserved if and only if it is holonomic.

Proof. The implication $\Rightarrow$ has been proved in Lemma 4.3.

We have $X^{\uparrow}[f]=X_{(1)}$ if and only if

$$
-\breve{G}_{0}^{i \sigma}\left(-\frac{1}{c_{0} \alpha^{0}} \partial_{\sigma} \breve{f}+X^{\rho} \partial_{\rho} \breve{G}_{0 \sigma}^{0}+\breve{G}_{0 \rho}^{0} \partial_{\sigma} X^{\rho}+\frac{1}{c_{0} \alpha^{0}} X^{\rho} \widehat{F}_{\rho \sigma}\right) \partial_{i}^{0}=\breve{\delta}_{\rho}^{i} \breve{\delta}_{0}^{\sigma} \partial_{\sigma} X^{\rho} \partial_{i}^{0} .
$$

But we can write $\breve{\delta}_{\rho}^{i}=\breve{G}_{0}^{i \sigma} G_{\sigma \rho}^{0}$ which imply that the above equation is equivalent to

$$
-\frac{1}{c_{0} \alpha^{0}} \breve{G}_{0}^{i \sigma}\left(-\partial_{\sigma} \breve{f}+X^{\rho} \widehat{F}_{\rho \sigma}+c_{0} \alpha^{0} \breve{\delta}_{0}^{\tau}\left(X^{\rho} \partial_{\rho} G_{\tau \sigma}^{0}+G_{\tau \rho}^{0} \partial_{\sigma} X^{\rho}+G_{\sigma \rho}^{0} \partial_{\tau} X^{\rho}\right)\right) \partial_{i}^{0}=0 .
$$

Now, if we consider the splitting $T^{*} \mathcal{J}_{1} \boldsymbol{E}=H_{\tau}^{*} \mathcal{J}_{1} \boldsymbol{E} \oplus V_{\gamma}^{*} \mathcal{J}_{1} \boldsymbol{E}$, we get, from the identity $\breve{G}_{i \rho}^{0} \breve{G}_{0}^{i \sigma}=\delta_{\rho}^{\sigma}-c \alpha^{0} \breve{\delta}_{0}^{\sigma} \tau_{\rho}$

$$
\partial_{\sigma} \breve{f}-X^{\rho} \widehat{F}_{\rho \sigma}=c_{0} \alpha^{0} \breve{\delta}_{0}^{\tau}\left(X^{\rho} \partial_{\rho} G_{\tau \sigma}^{0}+G_{\tau \rho}^{0} \partial_{\sigma} X^{\rho}+G_{\sigma \rho}^{0} \partial_{\tau} X^{\rho}\right)+k \tau_{\sigma},
$$

where $k: \mathfrak{\jmath}_{1} \boldsymbol{E} \rightarrow \mathbb{T}^{*}$ given as $k=$ д. $\left.\breve{f}-(X\lrcorner \widehat{F}\right)\left(\right.$ д) $-\left(L_{X} G\right)($ д, д) .

Now, let us suppose that $f$ be holonomic. By Proposition 4.2 we get

$$
d \breve{f}-X\lrcorner \widehat{F}=(\text { д. } \breve{f}-(X\lrcorner \widehat{F})(\text { д) }) \tau
$$

which is possible if and only if $d \breve{f}-X\lrcorner \widehat{F}=0$, i.e. if and only if $f$ is conserved. QED

Of course, a metric special phase function $f$ is holonomic and conserved if and only if $d \breve{f}=X\lrcorner \widehat{F}$. In Lemma 4.3 we have proved that for a Killing vector field $X$ if a corresponding special phase function $f=-G($ д, $X)+\breve{f}$ is conserved then it is holonomic. Now we shall prove the equivalence.

4.7 Theorem. Let $f=-G($ д, $X)+\breve{f}$ be a metric special phase function. Then $f$ is conserved and holonomic if and only if $X$ is an infinitesimal symmetry of $\widehat{F}$.

Proof. A metric special phase function is conserved and holonomic if and only if $d \breve{f}=X\lrcorner \widehat{F}$, i.e. $X\lrcorner \widehat{F}$ is closed. Then, for closed $\widehat{F}, 0=d(X\lrcorner \widehat{F})=\frac{1}{2} L_{X} \widehat{F}$ and $X$ is an infinitesimal symmetry of $\widehat{F}$.

On the other hand, if $X$ is a Killing vector field which is an infinitesimal symmetry of $\widehat{F}$, then $\left.0=L_{X} \widehat{F}=2 d(X\lrcorner \widehat{F}\right)$ and (locally) $\left.X\right\lrcorner \widehat{F}=d \breve{f}$, where $\breve{f}$ is a spacetime function given up to a constant. Then $f=-G(д, X)+\breve{f}$ is a conserved and holonomic special phase function. QED $d f$.

We call $f \in \operatorname{spec}\left(\mathcal{J}_{1} \boldsymbol{E}, \mathbb{R}\right), f=-G($ д, $X)+\breve{f}$, self-holonomic if $i_{X_{(1)}} \Omega=$

4.8 Theorem. $f$ is a self-holonomic special function if and only if $f$ is conserved and holonomic. 
Proof. If $f$ is a self-holonomic function, then $f$ is conserved. Indeed, let $f$ be selfholonomic; then $\gamma \cdot f=i_{\gamma} d f=i_{\gamma} i_{X_{(1)}} \Omega=-i_{X_{(1)}} i_{\gamma} \Omega=0$. Moreover, $X^{\uparrow}[f]=\gamma(\tau(X))+$ $d f^{\sharp}=\gamma(\tau(X))+\Lambda^{\sharp}\left(\Omega^{b}\left(X_{(1)}\right)\right)=\gamma(\tau(X))+X_{(1)}-\gamma(\tau(X))=X_{(1)}$.

On the other hand let $f$ be a conserved special holonomic function. Then $i_{X_{(1)}} \Omega=i_{X^{\uparrow}[f]} \Omega=i_{\left(d f^{\sharp}+\gamma(\tau(X))\right.} \Omega=\left(\Omega^{b} \circ \Lambda^{\sharp}\right)(d f)=d f-\gamma(d f) \tau=d f-(\gamma \cdot f) \tau=d f$. QED

4.9 Corollary. Let $X$ be a Killing vector field and $f=-G($ д, $X)+\breve{f}$ a corresponding special phase function. Then the following conditions are equivalent:

1) $f$ is conserved; 2) $f$ is holonomic; $\quad 3) f$ is self-holonomic.

4.10 Theorem. 1) Let $f=-G($ д, $X)+\breve{f}$ be a self-holonomic special phase function. Then, $X_{(1)}$ is an infinitesimal symmetry of $\Omega$.

2) Let $X$ be a vector field on $\boldsymbol{E}$ such that $X_{(1)}$ is an infinitesimal symmetry of $\Omega$. Then there exists a unique (up to a constant) self-holonomic special phase function $f$ such that $X_{(1)}=X^{\uparrow}[f]$.

Proof. 1) Let $f=-G($ д, $X)+\breve{f}$ be a self-holonomic special phase function, i.e. $i_{X_{(1)}} \Omega=d f$. Then $L_{X_{(1)}} \Omega=d i_{X_{(1)}} \Omega=d d f=0$.

2) Let $X_{(1)}$ is an infinitesimal symmetry of $\Omega$. Then by Theorem $3.13 X_{(1)}$ is of the form $X_{(1)}=\gamma(\tau(X))+d f^{\sharp}$, where $f$ is a conserved phase function uniquely given up to a constant. Projectability on $X$ then implies that $f$ has to be a special phase function $f=-G($ д, $X)+\breve{f}$. Moreover, we have $X_{(1)}=X^{\uparrow}[f]$. QED

4.11 Proposition. The subsheaf of self-holonomic special phase functions is closed with respect to the special bracket, i.e. if $f, h$ are selfholonomic, with $f=-G($ д, $X)+\breve{f}, h=-G\left(\right.$ д, $\left.X^{\prime}\right)+\breve{h}$, then

$$
i_{\left[X, X^{\prime}\right]_{(1)}} \Omega=d \llbracket f, h \rrbracket .
$$

Proof. We have $\gamma \cdot f=\gamma \cdot h=0$ and $X_{(1)}=X^{\uparrow}[f], X_{(1)}^{\prime}=X^{\uparrow}[h]$. To prove that $i_{\left[X, X^{\prime}\right]_{(1)}} \Omega=d \llbracket f, h \rrbracket$ it is sufficient to prove that $\left(\Lambda^{\sharp} \circ \Omega^{b}\right)\left(\left[X, X^{\prime}\right]_{(1)}\right)=\Lambda^{\sharp}(d \llbracket f, h \rrbracket)$ and $i_{\gamma} i_{\left[X, X^{\prime}\right]_{(1)}} \Omega=i_{\gamma} d \llbracket f, h \rrbracket$. We have

$$
\begin{aligned}
\left(\Lambda^{\sharp} \circ \Omega^{b}\right)\left(\left[X, X^{\prime}\right]_{(1)}\right) & =[X, Y]_{(1)}-\tau\left(\left[X, X^{\prime}\right]\right) \gamma=\left[X_{(1)}, X_{(1)}^{\prime}\right]-\tau\left(\left[X, X^{\prime}\right]\right) \gamma \\
& =\left[X^{\uparrow}[f], X^{\prime \uparrow}[h]\right]-\tau\left(\left[X, X^{\prime}\right]\right) \gamma=X^{\uparrow}[\llbracket f, h \rrbracket]-\tau\left(\left[X, X^{\prime}\right]\right) \gamma \\
& =\Lambda^{\sharp}(d \llbracket f, h \rrbracket)+\tau\left(\left[X, X^{\prime}\right]\right) \gamma-\tau\left(\left[X, X^{\prime}\right]\right) \gamma .
\end{aligned}
$$

Further we have $i_{\gamma} i_{\left[X, X^{\prime}\right]_{(1)}} \Omega=-i_{\left[X, X^{\prime}\right]_{(1)}} i_{\gamma} \Omega=0$, and

$$
i_{\gamma} d \llbracket f, h \rrbracket=i_{\gamma} d\{f, h\}=\{\gamma \cdot f, h\}+\{f, \gamma \cdot h\}=0 . \mathrm{QED}
$$




\subsection{Characterization of infinitesimal symmetries of the classical phase structure}

We shall start with infinitesimal symmetries of the contact gravitational phase structure given by the pair $\left(-\widehat{\tau}, \Omega^{\mathfrak{g}}\right)$.

4.12 Theorem. All projectable infinitesimal symmetries of the classical contact gravitational structure are, equivalently:

1. holonomic lifts of Killing vector fields;

2. vector fields $Y=(d \widehat{\tau}(X))^{\sharp}+\widehat{\tau}(X) \widehat{\gamma}^{\mathfrak{g}}$, where $X$ is a spacetime vector field such that $\widehat{\gamma}^{\mathfrak{g}} \cdot(\widehat{\tau}(X))=0$.

Proof. Item 1 follows from Theorem 3.7 and Proposition 3.10 Item 2 has been proved in 13. However, this statement follows from the equivalence between item 1 and item 2. Indeed, it is enough to observe that $\widehat{\gamma}^{\mathfrak{g}} \cdot \widehat{\tau}(X)=-\left(1 / c^{2}\right)\left(L_{X} g\right)($ д, Д). This implies the equivalence. QED

Now, let us assume the total almost-cosymplectic-contact pair $(-\widehat{\tau}, \Omega=$ $\Omega[G, F])$.

4.13 Theorem. All projectable infinitesimal symmetries of the classical almost-cosymplectic-contact structure $(-\widehat{\tau}, \Omega)$ are, equivalently:

1. special Hamiltonian lifts of (conserved) special phase functions $f=$ $-G($ д, $X)+\breve{f}$, where $X$ is a Killing vector field of $\boldsymbol{E}$ and $d \breve{f}=X\lrcorner \widehat{F}$;

2. vector fields $Y=d f^{\sharp}+\widehat{\tau}(X) \widehat{\gamma}$ where $f$ is a (conserved) special phase function $f=-G($ д, $X)+\breve{f}$ such that $\widehat{\gamma} \cdot f=0$ and $-i_{d f^{\sharp}} \Omega^{\mathfrak{g}}-\widehat{\tau}(X) i_{\widehat{\gamma}^{\mathfrak{c}}} \Omega^{\mathfrak{g}}+$ $d(\widehat{\tau}(X))=0$ is satisfied.

Proof. From Theorem 3.7 it follows that infinitesimal symmetries of $\widehat{\tau}$ are holonomic lifts $X_{(1)}$ of Killing vector fields of $\boldsymbol{E}$. By Theorem $4.10 X_{(1)}$ are infinitesimal symmetries of $\Omega$ if and only if they are special Hamiltonian lifts of self-holonomic (metric) special phase functions. Item 1 now follows from Theorem 4.7 Item 2 has been proved in [13, but it also follows from the equivalence of the two statements. The equivalence follows from the fact that for conserved special phase functions the condition 2 can be reduced to $-d \breve{f}+X\lrcorner \widehat{F}=0$. In fact, $\left.i_{\widehat{\gamma}^{\mathfrak{e}}} \Omega^{\mathfrak{g}}=-\widehat{\mathrm{A}}\right\lrcorner \widehat{F}$ and

$$
\begin{aligned}
i_{d f \sharp} \Omega^{\mathfrak{g}} & =\left(\Omega^{\mathfrak{g}}\right)^{\mathfrak{b}} \circ\left(\Lambda^{\mathfrak{g}}+\Lambda^{\mathfrak{e}}\right)^{\sharp}(d(\widehat{\tau}(X))+d \breve{f}) \\
& =d(\widehat{\tau}(X))+d \breve{f}-\widehat{\gamma}^{\mathfrak{g}}(d(\widehat{\tau}(X))+d \breve{f}) \widehat{\tau}+\left(\Omega^{\mathfrak{g}}\right)^{\mathfrak{b}} \circ\left(\Lambda^{\mathfrak{e}}\right)^{\sharp}(d(\widehat{\tau}(X))) .
\end{aligned}
$$

But from $\widehat{\gamma} \cdot f=0$ we have $\widehat{\gamma}^{\mathfrak{g}}(d(\widehat{\tau}(X))+d \breve{f})=-\widehat{\gamma}^{\mathfrak{e}}(d(\widehat{\tau}(X))+d \breve{f})=-\widehat{\gamma}^{\mathfrak{e}}(d(\widehat{\tau}(X)))=$ $\widehat{\mathbb{M}}\lrcorner(X\lrcorner \widehat{F})$. Moreover, $\left.\left.\left.\left.\left(\Omega^{\mathfrak{g}}\right)^{\mathfrak{b}} \circ\left(\Lambda^{\mathfrak{e}}\right)^{\sharp}(d(\widehat{\tau}(X)))=-X\right\lrcorner \widehat{F}+(\widehat{\mathbb{\mu}}\lrcorner \widehat{F}\right) \widehat{\tau}(X)+\widehat{\mathbb{\mu}}\right\lrcorner(X\lrcorner \widehat{F}\right) \widehat{\tau}$, from which the equivalence follows. QED 
4.14 Corollary. Infinitesimal symmetries of the classical structure are special Hamiltonian lifts $X^{\uparrow}[f]$ of special phase functions $f=-G($ д, X) + $\breve{f}$, where $L_{X} G=0$ and $\left.d \breve{f}=X\right\lrcorner \widehat{F}$. In this case $X^{\uparrow}[f]=X_{(1)}$ and the coordinate expression of infinitesimal symmetries of the classical structure are $X_{(1)}=X^{\lambda} \partial_{\lambda}+\breve{\delta}_{\rho}^{i} \breve{\delta}_{0}^{\sigma} \partial_{\sigma} X^{\rho} \partial_{i}^{0}$, where $\partial_{\lambda} \breve{f}=X^{\rho} \widehat{F}_{\rho \lambda}$ and $X^{\rho} \partial_{\rho} G_{\lambda \mu}^{0}+$ $G_{\rho \mu}^{0} \partial_{\lambda} X^{\rho}+G_{\lambda \rho}^{0} \partial_{\mu} X^{\rho}=0$.

4.15 Theorem. Special phase functions which are generators of phase infinitesimal symmetries of the classical structure form a Lie algebra with respect to the special bracket.

Proof. By Theorem 4.13 generators of infinitesimal symmetries of the classical structure are self-holonomic metric special phase functions. Theorem now follows from Proposition 4.11, QED

Note that for a Killing vector field $X$ the generator of the infinitesimal symmetry $X^{\uparrow}[f]$ of the classical structure is a pair $(X, \breve{f})$, where $\breve{f}$ is a spacetime function satisfying $d \breve{f}=X\lrcorner \widehat{F}$, i.e. $\breve{f}$ is given up to a constant.

\subsection{Momentum map and special phase functions}

In this subsection, we will extend the theory of momentum map from symplectic geometry (see [30]) to our almost-cosymplectic-contact geometry. The extension mimick previous more general theories for both contact and cosymplectic geometry [1, 4, 6]. We prove that our model always admits a momentum map.

Let $G$ be a Lie group and $\Phi: G \times \mathcal{J}_{1} \boldsymbol{E} \rightarrow \mathcal{J}_{1} \boldsymbol{E}$ be a left action such that for each $g \in G \Phi_{g}^{*} \widehat{\tau}=\widehat{\tau}$ and $\Phi_{g}^{*} \Omega=\Omega$. The associated infinitesimal action $\phi: \mathfrak{g} \times$ $\mathcal{J}_{1} \boldsymbol{E} \rightarrow T \mathcal{J}_{1} \boldsymbol{E}$, where $\phi(\xi)_{j_{1} s(x)}=-T \Phi_{j_{1}(x)}(\xi)$ is an action by infinitesimal symmetries of the classical almost-cosymplectic-contact structure $(-\widehat{\tau}, \Omega)$. If we suppose $\Phi$ or $\phi$ to be projectable to actions $\bar{\Phi}$ and $\bar{\phi}$ on $\boldsymbol{E}$, then by Theorem 4.13 for each $\xi \in \mathfrak{g}$ we have $\phi(\xi)=\bar{\phi}(\xi)_{(1)}$, where $\bar{\phi}(\xi)$ is a Killing vector field which is also a symmetry of the electromagnetic field $F$.

A momentum map for a projectable action $\Phi$ of a Lie group $G$ of infinitesimal symmetries of the classical almost-cosymplectic-contact structure is a map $J: \mathcal{J}_{1} \boldsymbol{E} \rightarrow \mathfrak{g}^{*}$ such that $i_{\phi(\xi)} \Omega=-d J_{\xi}$, where $J_{\xi}: \mathcal{J}_{1} \boldsymbol{E} \rightarrow \mathbb{R}$. By Theorem $4.8 J_{\xi}$ is a conserved special function. A momentum map $J$ is equivariant if for all $g \in G J \circ \Phi_{g}=\mathrm{CoAd}_{g} \circ J=J_{\mathrm{Ad}_{g^{-1}}}$, where Ad is the adjoint action on $\mathfrak{g}$ and CoAd is the coadjoint action on $\mathfrak{g}^{*}$. There are several obstructions for the existence of the momentum map in general. However, our model always admits a momentum map on the domain of the Poincaré-Cartan form $\Theta$. 
4.16 Proposition. Let $\Phi$ be a projectable action of a Lie group $G$ of infinitesimal symmetries of the classical almost-cosymplectic-contact structure. Then, the map $J$ defined by $J_{\xi}=i_{\phi(\xi)} \Theta$ is an equivariant momentum map by conserved special functions for $\Phi$. indeed,

Proof. It is easy to prove that $-d\left(i_{\phi(\xi)} \Theta\right)=i_{\phi(\xi)} \Omega$. This map is also equivariant:

$$
\begin{array}{r}
J_{\mathrm{Ad}_{g^{-1}} \xi}\left(j_{1} s(x)\right)=\Theta\left(\operatorname{Ad}_{g^{-1}} \xi\right)\left(j_{1} s(x)\right)=\Theta\left(T \Phi_{g^{-1}} \phi(\xi)\right)\left(j_{1} s(x)\right)= \\
=\Phi_{g}^{*} \Theta\left(T \Phi_{g^{-1}} \phi(\xi)\right)\left(j_{1} s(x)\right)=J_{\xi}\left(\Phi_{g}\left(j_{1} s(x)\right)\right) . \text { QED }
\end{array}
$$

\subsection{Examples}

Here we will briefly discuss momentum maps for symmetry groups acting on Minkowski and Reissner-Nordstrom spacetimes.

Minkowski spacetime. The Poincare group is acting on Minkowski spacetime as its group of isometries. Let us denote by $\mathfrak{p}$ its Lie algebra. After the introduction of adapted linear coordinates on $\boldsymbol{E}, \mathfrak{p}$ acts on $\boldsymbol{E}$ in the standard affine way. As is well-known, $\mathfrak{p}$ is the semi-direct product of $\mathbb{R}^{4}$ (representing translations) and $\mathfrak{s o}(1,3)$ (representing Lorentz transformations). The momentum map takes the form

$$
J_{\xi}=i_{\phi(\xi)}(-\widehat{\tau})=(m c / \hbar) \alpha^{0}\left(-\bar{\phi}(\xi)^{0}+x_{0}^{1} \bar{\phi}(\xi)^{1}+x_{0}^{2} \bar{\phi}(\xi)^{2}+x_{0}^{3} \bar{\phi}(\xi)^{3}\right),
$$

where $\bar{\phi}$ is the infinitesimal action on spacetime. In particular, when $\xi=$ $e_{i} \in \mathbb{R}^{4}$, then $\bar{\phi}(\xi)=\partial_{i}$ and $J_{\xi}=(m c / \hbar) \alpha^{0} x_{0}^{i}$; when $\xi$ is a spacelike rotation then $\bar{\phi}(\xi)=x^{i} \partial_{j}-x^{j} \partial_{i}$ and $J_{\xi}=(m c / \hbar) \alpha^{0}\left(x^{i} x_{0}^{j}-x^{j} x_{0}^{i}\right)$.

Reissner-Nordstrom spacetime. This spacetime is obtained by requirements of spherical symmetry so its infinitesimal isometries are rotations and, since the metric is static, time translations. The Lie algebra of the corresponding Lie group is the semidirect product of $\mathbb{R}$ and $\mathfrak{s o}(3)$. The momentum map turns out to be

$$
J_{\xi}=i_{\phi(\xi)}(-\widehat{\tau}+\widehat{A}),
$$

where $\widehat{A}$ was given in (21) and $\tau$ in (23). Now, when $\xi$ corresponds to time translation we have $\bar{\phi}(\xi)=\partial / \partial t$ and $J_{\xi}=(m c / \hbar) \alpha^{0}\left(-\left(1-k_{s} / r+k_{q}^{2} / r^{2}\right)-\right.$ $\left.\left(q_{0} /\left(\hbar_{0} r\right)\right)\right)$, which is the Hamiltonian; when $\xi$ corresponds to spacelike rotations we convert the vector field $\bar{\phi}(\xi)=x^{i} \partial_{j}-x^{j} \partial_{i}$ to spherical coordinates then contract it with $-\widehat{\tau}+\widehat{A}$. We obtain a long coordinate expression which is not difficult to compute and to which there is no contribution from $\widehat{A}$. 


\section{$5 \quad$ Perspectives}

We characterized infinitesimal phase symmetries of spacetime starting from a quite natural Definition 3.4. Various properties of such symmetries have been derived, the most promising one coming from Proposition 4.16. The Proposition opens the possibility to reduce the almost-cosymplectic-contact structure along the lines of [1, 4, 6] (which are inspired by the classical MarsdenWeinstein reduction). Namely, since motions happen on level surfaces of the form $J^{-1}(\mu)$ it is natural to restrict the dynamical system to $J^{-1}(\mu)$. Then it could be proved that the resulting almost-cosymplectic-contact structure would be degenerate along directions tangent to group orbits in $J^{-1}(\mu)$. A quotient by the stabilizer of $\mu$ in $\mathfrak{g}^{*}$ should yield a new almost-cosymplecticcontact structure for the reduced dynamical system. As far as we know, this reduction has never been attempted in relativistic mechanics.

Another direction of investigation is given by enlarging the class of symmetries under consideration. The requirement of projectability is quite natural under the physical viewpoint, but it could be dropped in order to consider symmetries of the generalized contact structure which depend on phase space in an essentially non-projectable way, as we already mentioned in the Introduction. Noether symmetries of non-projectable type correspond to conserved quantities that, in the standard 4-velocity formalism, depend polynomially on velocities 33 . The coefficients of monomials are Killing tensors. This construction could be repeated in the framework of our model. KillingYano tensors are also related with non-projectable symmetries through a similar mechanism.

The task of our research has been the systematic exploration of infinitesimal symmetries of the geometric structures on the general relativistic phase space. The idea that we had in mind was to do a symmetry analysis in the framework of generalized contact geometry. We pursued this task by characterizing projectable infinitesimal symmetries of the generalized contact structure and by relating such symmetries with a distinguished class of observables on the phase space, the special phase functions. Moreover, we were able to relate brackets of symmetries with brackets of special phase functions. Finally, we proved that a momentum map for a group actions by projectable infinitesimal symmetries always exist. Now, the most natural question that we can ask ourselves is if it is possible to generalize the above results to the case of non-projectable infinitesimal symmetries. At the present moment, we cannot predict which of the results in the paper could be extended to more general symmetries. In particular, we do not know which class of conserved quantities would correspond to non-projectable infinitesimal symmetries and if we would be able to provide a bracket on such an 
unknown class; we also ignore if a momentum map could be introduced for such symmetries. Maybe it would be possible to introduce a distinguished class of non-projectable symmetries that would be in correspondence with Killing and/or Killing-Yano tensors, and find corresponding observables, a bracket between them etc.. The starting point would be exactly relaxing the hypotheses of Theorem 3.7 in order to allow for non-projectable symmetries. This will be the subject of future work.

\section{References}

[1] C. Albert: Le théorème de reduction de Marsden-Weinstein en géométrie cosymplectique et de contact, J. Geom. Phys. 6 (1989), 627-649.

[2] D. V. Alekseevsky, V. V. Lychagin, A. M. Vinogradov: Basic ideas and concepts of differential geometry, Geometry I. Encycl. Math. Sci. 28, Springer-Verlag, Berlin, 1991.

[3] A. V. Bocharov, V. N. Chetverikov, S. V. Duzhin, N. G. Khor'kova, I. S. Krasil'shchik, A. V. Samokhin, Yu. N. Torkhov, A. M. Verbovetsky and A. M. Vinogradov: Symmetries and Conservation Laws for Differential Equations of Mathematical Physics, I.S. Krasil'shchik and A.M. Vinogradov eds., Translations of Math. Monographs 182, Amer. Math. Soc. (1999).

[4] F. Cantrijn, J. CorTes: Cosymplectic reduction of constrained systems with symmetry, Rep. Math. Phys. 49 (2002), 167-182.

[5] P. Dedecker: On applications of homological algebra to calculus of variations and mathematical physics, Proceedings of the IV international colloquium on differential geometry, Santiago de Compostela, Universidad de Santiago de Compostela, Cursos y Congresos de la Universidad de Santiago de Compostela 15 (1978), 285-294.

[6] M. DE LeOn, M. SARALEgi: Cosymplectic reduction for singular momentum maps, J. Phys. A 26 (1993), 5033-5043.

[7] M. De Leon, G.M. Tuynman: A universal model for cosymplectic manifolds, J. Geom. Phys. 20 (1996) 77-86.

[8] G. Fülop, D. M. Gitman, I. V. Tyutin: Reparametrization Invariance as Gauge Symmetry, Internat. J. Theoret. Phys. 38(1999), 1941-1968.

[9] G.S. HALL: Symmetries and curvature structure in general relativity, World Scientific Lecture Notes in Physics 46, 2004.

[10] M. Henneaux, C. Teitelboim: Quantization of Gauge Systems, Princeton Univ. Press 1992.

[11] T. IwAI: Symmetries in relativistic dynamics of a charged particle, Ann. Inst. H. Poincaré Sect. A (N.S.) 25 (1976), 335-343. 
[12] J. JANYŠKA:: Natural Lagrangians for quantum structures over 4-dimensional spaces, Rend. di Mat., S VII, Vol 18, Roma (1998), 623-648.

[13] J. JANYŠKA: Special phase functions and phase infinitesimal symmetries in classical general relativity, AIP Conf. Proc. 1460, XX Internat. Fall Workshop on Geometry and Physics, 135-140.

[14] J. JAnYŠKa, M. Modugno:: Classical particle phase space in general relativity, in: Differential Geometry and Applications, Proc. Conf., Aug. 28 - Sept. 1, 1996, Brno, Czech republic, Masaryk University, Brno 1996, 573-602.

[15] J. JANYŠKA, M. Modugno: Hermitian vector fields and special phase functions, Int. J. Geom. Methods Mod. Phys. 3 (2006), 719-754.

[16] J. JanyšKa, M. Modugno: Geometric Structures of the Classical General Relativistic Phase Space, Int. J. Geom. Methods Mod. Phys. 5 (2008), 699-754.

[17] J. JAnYŠKA, M. Modugno: Generalized geometrical structures of odd dimensional manifolds, J. Math. Pures Appl. (9) 91 (2009), 211-232.

[18] J. JANYŠKA, M. Modugno: Special bracket on the classical general relativistic phase space, preprint 2012.

[19] J. JAnyŠKa, M. Modugno, D. SAller: Covariant quantum mechanics and infinitesimal quantum symmetries, preprint 2011.

[20] J. Janyška, M. Modugno, R. Vitolo: An Algebraic Approach to Physical Scales, Acta Appl. Math. 110 (2010), 1249-1276.

[21] I. Kolář, P. Michor, J. Slovák: Natural Operations in Differential Geometry, Springer-Verlag, 1993.

[22] P. Libermann, Ch. M. Marle: Symplectic Geometry and Analytical Mechanics, Reidel Publ., Dordrecht, 1987.

[23] A. Lichnerowicz: Les varietés de Jacobi et leurs algèbres de Lie associées,, J. Math. Pures Appl. 57 (1978) 453-488.

[24] G. Manno, R. Vitolo: Relativistic mechanics, contact manifolds and symmetries, Note Mat., 23 (2004/2005), 157-171.

[25] G. Manno, R. Vitolo: Geometric aspects of higher order variational principles on submanifolds, Acta Appl. Math. 101 (2008), 215-229.

[26] M. Modugno, D. Saller, J. Tolksdorf: Classification of infinitesimal symmetries in covariant classical mechanics, J. Math. Phys. 47 (2006), 1-27.

[27] M. Modugno, A. M. Vinogradov: Some variations of the notion of connection, Ann. Mat. Pura Appl. (4) 167 (1994), 33-71.

[28] P. A. Nikolov, I. T. Todorov: Space-time description of motion and the Hamiltonian approach to the dynamics of relativistic particles, Sov. J. Part. Nucl. 14 (1983), 458-466. 
[29] P. Olver: Applications of Lie groups to differential equations, Graduate Texts in Mathematics 107, 2nd edit., Springer 1992.

[30] J.-P. Ortega, T. Ratiu: Momentum Maps and Hamiltonian Reduction, Progress in Mathematics 222, Birkhäuser, 2004.

[31] Y. S. Poon, A. WAde: Generalized contact structures, J. London Math. Soc. (2) 83 (2011), 333-352.

[32] D. Saller, R. Vitolo: Symmetries in covariant classical mechanics, J. Math. Phys. 41 (2000), 6824-6842.

[33] O.P. Santillan: Hidden symmetries and supergravity solutions, J. Math. Phys. 53 (2012), 043509.

[34] G. A. Sardanashvily: Hamiltonian time-dependent mechanics, J. Math. Phys. 39 (1998), 2714-2729.

[35] A.M. Vinogradov: A spectral sequence associated with a non-linear differential equation, and algebro-geometric foundations of Lagrangian field theory with constraints, Soviet Math. Dokl. 19 (1978), 144-148.

[36] R. Vitolo: Quantum structures in Einstein general relativity, Lett. Math. Phys., 51 (2000), 119-133.

[37] A. WADE: Local structure of generalized contact manifolds, Diff. Geom. Appl. 30 (2012), 124-135. 